\title{
HIGH-ORDER PHASE TRANSITIONS IN THE QUADRATIC FAMILY
}

\author{
DANIEL CORONEL AND JUAN RIVERA-LETELIER
}

\begin{abstract}
We give the first example of a transitive quadratic map whose real and complex geometric pressure functions have a high-order phase transition. In fact, near the phase transition these functions behave as

$$
x \mapsto \exp \left(-x^{-2}\right)
$$

near $x=0$, before becoming linear. This quadratic map has a non-recurrent critical point, so it is non-uniformly hyperbolic in a strong sense.
\end{abstract}

\section{INTRODUCTION}

This paper is concerned with the thermodynamic formalism of smooth dynamical systems. Such a study was initiated by Sinai, Ruelle, and Bowen Sin72, Bow75, Rue76, in the context of uniformly hyperbolic diffeomorphisms and Hölder continuous potentials. In the last decades there has been important efforts to extend the theory beyond the uniformly hyperbolic setting, specially in real and complex dimension 1 where a complete picture is emerging, see for example [BT09, MS00, MS03, PS08, PRL11, PRL13] and references therein. See also [Sar11, UZ09, VV10] and references therein for (recent) results in higher dimensions.

For a smooth map $f$ in real or complex dimension 1 and a real parameter $t$, we consider the pressure of $f$ with respect to the geometric potential $-t \log |D f|$, see $\$ 1.1$ for precisions. The function of $t$ so defined is the geometric pressure function of $f$. It is closely related to several multifractal spectra and large deviation rate functions associated with $f$, see for example BMS03, Lemma 2], GPR10, [T11, KN92, Theorems 1.2 and 1.3], PRL11, Appendix B], and references therein.

We exhibit a transitive quadratic map whose geometric pressure function behaves, for some constants $A>0$ and $\chi>0$ and for $t$ near a certain parameter $t_{*}$, as the function

$$
t \mapsto \begin{cases}-t \chi+\exp \left(-A\left(t_{*}-t\right)^{-2}\right) & \text { if } t<t_{*} ; \\ -t \chi & \text { if } t \geq t_{*},\end{cases}
$$

see the Main Theorem in $\$ 1.1$ In particular, the geometric pressure function of this map is not real analytic at $t=t_{*}$; that is, it has a phase transition at $t=t_{*}$ in the sense of statistical mechanics. This is the first example of a transitive smooth dynamical system having a phase transition of infinite contact order. This example is also robust: Every family of sufficiently regular unimodal maps that is close to the quadratic family has a member with the same property.

The quadratic map we study has a non-recurrent critical point, so it is nonuniformly hyperbolic in a strong sense. Thus, roughly speaking, lack of expansion is not responsible for the phase transition. Instead, it is the irregular behavior of the critical orbit that is one of the mechanisms behind the phase transition. Considering 
a different behavior of the critical orbit, in the companion paper [CRL12] we gave the first example of a quadratic map having a phase transition at a large value of $t$; that is, of a "low-temperature phase transition", see also [MS03, §5] for some conformal Cantor sets with similar properties. In contrast with the example studied here, the geometric pressure function of the quadratic map studied in CRL12 is not differentiable at the phase transition; that is, it is a phase transition of first order.

Another interesting feature of the quadratic map we study is that it has no equilibrium state at the phase transition. At a low-temperature phase transition there can be at most 1 equilibrium state $*$ and in the companion paper CRL12] we provide an example of a quadratic map having one.

There are various examples in the literature of transitive smooth maps whose geometric pressure function has a first-order phase transition. This includes quadratic maps that have an absolutely continuous invariant measure, and that do not satisfy the Collet-Eckmann condition 1 By the work of Makarov and Smirnov [MS00, this also includes those phase transitions in the complex case that occur at a parameter in $(-\infty, 0)$. See also DGR11, DGR13, LOR11, for examples of first-order phase transitions of some transitive 3-dimensional diffeomorphisms.

Another type of phase transition that has been studied in detail, is that related to the existence of a neutral periodic point. For a given $\alpha \geq 1$, Lopes shows in Lop93 that for $t<1$ close to 1 the geometric pressure function of the map $f_{\alpha}$ given by $x \mapsto x\left(1+x^{\alpha}\right) \bmod 1$, is of the order of $(1-t)^{\alpha}$; on the other hand, this function is identically zero on $[1,+\infty)$. In view of this result, it is expected that for a quadratic map $f$ having a periodic point $p$ of period $n \geq 1$ satisfying $D f^{n}(p)= \pm 1$, the geometric pressure function of $f$ has a unique phase transition, and that this phase transition is of finite order

1.1. Statement of results. We consider a set of real parameters $c$ close to -2 , such that $f_{c}(c)>c$, such that the interval $I_{c}:=\left[c, f_{c}(c)\right]$ of $\mathbb{R}$ is invariant by $f_{c}$, and such that $f_{c}$ is topologically exact on this set. We consider 2 dynamical systems associated to $f_{c}$ : The interval map $\left.f_{c}\right|_{I_{c}}$, and the complex quadratic polynomial $f_{c}$ acting on its Julia set $J_{c}$.

For such $c$, define

$$
\chi_{\text {crit }}(c):=\liminf _{m \rightarrow+\infty} \frac{1}{m} \log \left|D f_{c}^{m}(c)\right|,
$$

and denote by $\mathscr{M}_{c}^{\mathbb{R}}$ the space of Borel probability measures supported on $I_{c}$ that are invariant by $f_{c}$. For a measure $\mu$ in $\mathscr{M}_{c}^{\mathbb{R}}$ denote by $h_{\mu}\left(f_{c}\right)$ the measure-theoretic entropy of $f_{c}$ with respect to $\mu$ and for each $t$ in $\mathbb{R}$ put

$$
P_{c}^{\mathbb{R}}(t):=\sup \left\{h_{\mu}\left(f_{c}\right)-t \int \log \left|D f_{c}\right| d \mu \mid \mu \in \mathscr{M}_{c}^{\mathbb{R}}\right\},
$$

${ }^{*}$ See [Dob13 Theorem 6] in the real setting, and [Dob12 Theorem 8] in the complex setting.

${ }^{\dagger}$ For such a map, the geometric pressure function is identically zero after its first zero, see NS98, Theorem A] or RL12, Corollary 1.3] in the real case and [PRLS03, Main Theorem] in the complex case. On the other hand, since every absolutely continuous invariant measure has a strictly positive Lyapunov exponent, the existence of such a measure easily implies that the geometric pressure function is not differentiable at its first zero.

${ }^{\ddagger}$ Notice that, when $D f^{n}(p)=1$ (resp. $D f^{n}(p)=-1$ ), the function $x \mapsto f^{n}(x)-x$ (resp. $\left.x \mapsto f^{2 n}(x)-x\right)$ is of the order of $(x-p)^{2}\left(\right.$ resp. $\left.(x-p)^{3}\right)$ near $p$, see for example [CG93, Mil06]. 
which is finite. The function $P_{c}^{\mathbb{R}}: \mathbb{R} \rightarrow \mathbb{R}$ so defined is called the geometric pressure function of $\left.f_{c}\right|_{I_{c}}$; it is convex and nonincreasing.

Similarly, denote by $\mathscr{M}_{c}^{\mathbb{C}}$ the space of Borel probability measures supported on $J_{c}$ that are invariant by $f_{c}$ and for a measure $\mu$ in $\mathscr{M}_{c}^{\mathbb{C}}$ we denote by $h_{\mu}\left(f_{c}\right)$ the measure-theoretic entropy of $f_{c}$ with respect to $\mu$. Then the geometric pressure function $P_{c}^{\mathbb{C}}: \mathbb{R} \rightarrow \mathbb{R}$ of $f_{c}$ is defined by

$$
P_{c}^{\mathbb{C}}(t):=\sup \left\{h_{\mu}\left(f_{c}\right)-t \int \log \left|D f_{c}\right| d \mu \mid \mu \in \mathscr{M}_{c}^{\mathbb{C}}\right\} .
$$

Main Theorem. There is a real parameter $c$ such that the critical point of $f_{c}$ is non-recurrent, such that for some $t_{*}>0$ and every $t \geq t_{*}$, we have

$$
P_{c}^{\mathbb{R}}(t)=P_{c}^{\mathbb{C}}(t)=-t \frac{\chi_{\text {crit }}(c)}{2},
$$

and such that for some constants $A>0, B^{+}>0$, and $B^{-}>0$, we have for every $t$ in $\left(0, t_{*}\right)$ close to $t_{*}$

$$
\begin{aligned}
-t \frac{\chi_{\mathrm{crit}}(c)}{2}+2^{-\left(\frac{A}{t_{*}-t}+B^{-}\right)^{2}} \leq P_{c}^{\mathbb{R}}(t) \leq P_{c}^{\mathbb{C}}(t) & \\
& \leq-t \frac{\chi_{\text {crit }}(c)}{2}+2^{-\left(\frac{A}{t_{*}-t}-B^{+}\right)^{2} .}
\end{aligned}
$$

In particular, both $P_{c}^{\mathbb{R}}$ and $P_{c}^{\mathbb{C}}$ are of class $C^{2}$ at $t=t_{*}$, but neither of these functions is real analytic at $t=t_{*}$.

We show in addition that there is no equilibrium state at the phase transition, that there is a unique associated conformal measure, and that this last measure is dissipative and purely atomic, see $\$ 3.1$ for definitions and for a strengthened version of the Main Theorem. It can also be shown that, if for each $t$ in $\left(0, t_{*}\right)$ we denote by $\nu_{t}$ the unique equilibrium state of $f_{c}$ for the potential $-t \log \left|D f_{c}\right|$, then the measure $\nu_{t}$ converges as $t \mapsto t_{*}^{-}$to the invariant probability measure supported on a certain periodic point of period 3 of $f_{c}$.

Since the critical point of a map $f_{c}$ as in the Main Theorem is non-recurrent, it follows that $f_{c}$ satisfies the Collet-Eckmann condition: $\chi_{\text {crit }}(c)>0$, see [Mis81] for the real case and Mañ93 for the complex case. So, $t_{*}$ in the Main Theorem is strictly larger than the first zero of the geometric pressure function of $f_{c}$; that is, $f_{c}$ has a "low-temperature" phase transition at $t=t_{*}$ in the sense of CRL12.

1.2. Notes and references. For complex rational maps, Makarov and Smirnov showed that every phase transition occurring at a negative parameter is removable, in the sense that the geometric pressure function has a real analytic continuation to all of $(-\infty, 0)$, see [MS00, Theorem B]. In contrast, the geometric pressure function of a map as in the Main Theorem cannot admit a real analytic continuation beyond the phase transition.

For a map as in the Main Theorem, the non-existence of equilibrium states also follows from IRRL12, Corollary 1.3].

For a quadratic map having a phase transition at the first zero of the pressure function, that is, a high-temperature phase transition, the number of ergodic equilibrium states can be arbitrary, see [CRL10, Corollaries 2 and 3], and also BK98, Example 5.4] and [BT06, Corollary 2] for an example having no equilibrium state.

Bruin and Todd study in BT12 certain piecewise linear models (with an infinite number of break points) of the smooth unimodal maps having a wild attractor 
in BKNvS96. They show that for a large value of the order of the critical point, the piecewise linear model has a high-order phase transition. Notice that no quadratic map can have a wild attractor, see Lyu94.

1.3. Strategy and organization. To prove the Main Theorem, we consider the set of parameters introduced in CRL12. For each parameter $c$ in this set, the critical value is eventually mapped to an expanding Cantor set, denoted by $\Lambda_{c}$. For such a parameter, the behavior of the geometric pressure function at low temperatures is intimately related to the derivatives of the map along the critical orbit (Proposition 5.6). As a first approximation we use the multipliers of the 2 periodic orbits of period 3 of $f_{c}$ to estimate these derivatives. However, the distortion constants in these estimates are too big to achieve the level of precision needed to prove the Main Theorem. To achieve a higher precision, we estimate these distortion constants in terms of the total distortion along certain homoclinic orbits connecting the 2 periodic orbits of period 3 (Proposition 3.1 in $\$ 3.2$ ).

We now proceed to describe the organization of the paper more precisely.

After some preliminaries in 92 , we state an strengthened version of the Main Theorem in 3.1 as the "Main Technical Theorem". In 3.3 we introduce an abstract 2 variables series that captures the behavior of the geometric pressure function at low temperatures (Proposition A). Its definition is based on an approximation of the derivatives at the critical value in terms of its itinerary in $\Lambda_{c}$ (Proposition 3.1 in (3.2), as mentioned above.

In which is independent of the rest of the paper, we study in an abstract setting the 2 variables series for an specific class of itineraries. We show that this series has a phase transition with an asymptotic behavior as in the Main Theorem. The itineraries are defined in 4 4.1, and the estimates of the corresponding 2 variables series are made in 4.2 ,

The proof of the Main Technical Theorem is given in $\$ 5$. After some general results about conformal measures in 95.1 , we make some technical estimates in 45.2 The proof of the Main Technical Theorem is in $\$ 5.3$, after recalling a few results from [CRL12].

1.4. Acknowledgments. The first named author acknowledges partial support from FONDECYT grant 11121453. This article was completed while second named author was visiting Brown University and the Institute for Computational and Experimental Research in Mathematics (ICERM). He thanks both of these institutions for the optimal working conditions provided, and acknowledges partial support from FONDECYT grant 1100922.

\section{Preliminaries}

We use $\mathbb{N}$ to denote the set of integers that are greater than or equal to 1 and $\mathbb{N}_{0}:=\mathbb{N} \cup\{0\}$.

2.1. Quadratic polynomials, Green functions, and Böttcher coordinates. In this subsection and the next we recall some basic facts about the dynamics of complex quadratic polynomials, see for instance CG93 or Mil06 for references.

For $c$ in $\mathbb{C}$ we denote by $f_{c}$ the complex quadratic polynomial

$$
f_{c}(z)=z^{2}+c,
$$


and by $K_{c}$ the filled Julia set of $f_{c}$; that is, the set of all points $z$ in $\mathbb{C}$ whose forward orbit under $f_{c}$ is bounded in $\mathbb{C}$. The set $K_{c}$ is compact and its complement is the connected set consisting of all points whose orbit converges to infinity in the Riemann sphere. Furthermore, we have $f_{c}^{-1}\left(K_{c}\right)=K_{c}$ and $f_{c}\left(K_{c}\right)=K_{c}$. The boundary $J_{c}$ of $K_{c}$ is the Julia set of $f_{c}$.

For a parameter $c$ in $\mathbb{C}$, the Green function of $K_{c}$ is the function $G_{c}: \mathbb{C} \rightarrow$ $[0,+\infty)$ that is identically 0 on $K_{c}$, and that for $z$ outside $K_{c}$ is given by the limit,

$$
G_{c}(z)=\lim _{n \rightarrow+\infty} \frac{1}{2^{n}} \log \left|f_{c}^{n}(z)\right|>0 .
$$

The function $G_{c}$ is continuous, subharmonic, satisfies $G_{c} \circ f_{c}=2 G_{c}$ on $\mathbb{C}$, and it is harmonic and strictly positive outside $K_{c}$. On the other hand, the critical values of $G_{c}$ are bounded from above by $G_{c}(0)$, and the open set

$$
U_{c}:=\left\{z \in \mathbb{C} \mid G_{c}(z)>G_{c}(0)\right\}
$$

is homeomorphic to a punctured disk. Notice that $G_{c}(c)=2 G_{c}(0)$, thus $U_{c}$ contains $c$.

By Böttcher's Theorem there is a unique conformal representation

$$
\varphi_{c}: U_{c} \rightarrow\left\{z \in \mathbb{C}|| z \mid>\exp \left(G_{c}(0)\right)\right\},
$$

and this map conjugates $f_{c}$ to $z \mapsto z^{2}$. It is called the Böttcher coordinate of $f_{c}$ and satisfies $G_{c}=\log \left|\varphi_{c}\right|$.

2.2. External rays and equipotentials. Let $c$ be in $\mathbb{C}$. For $v>0$ the equipotential $v$ of $f_{c}$ is by definition $G_{c}^{-1}(v)$. A Green's line of $G_{c}$ is a smooth curve on the complement of $K_{c}$ in $\mathbb{C}$ that is orthogonal to the equipotentials of $G_{c}$ and that is maximal with this property. Given $t$ in $\mathbb{R} / \mathbb{Z}$, the external ray of angle $t$ of $f_{c}$, denoted by $R_{c}(t)$, is the Green's line of $G_{c}$ containing

$$
\left\{\varphi_{c}^{-1}(r \exp (2 \pi i t)) \mid \exp \left(G_{c}(0)\right)<r<+\infty\right\} .
$$

By the identity $G_{c} \circ f_{c}=2 G_{c}$, for each $v>0$ and each $t$ in $\mathbb{R} / \mathbb{Z}$ the map $f_{c}$ maps the equipotential $v$ to the equipotential $2 v$ and maps $R_{c}(t)$ to $R_{c}(2 t)$. For $t$ in $\mathbb{R} / \mathbb{Z}$ the external ray $R_{c}(t)$ lands at a point $z$, if $G_{c}: R_{c}(t) \rightarrow(0,+\infty)$ is a bijection and if $\left.G_{c}\right|_{R_{c}(t)} ^{-1}(v)$ converges to $z$ as $v$ converges to 0 in $(0,+\infty)$. By the continuity of $G_{c}$, every landing point is in $J_{c}=\partial K_{c}$.

The Mandelbrot set $\mathcal{M}$ is the subset of $\mathbb{C}$ of those parameters $c$ for which $K_{c}$ is connected. The function

$$
\begin{aligned}
\Phi: \mathbb{C} \backslash \mathcal{M} & \rightarrow \mathbb{C} \backslash \operatorname{cl}(\mathbb{D}) \\
c & \mapsto \Phi(c):=\varphi_{c}(c)
\end{aligned}
$$

is a conformal representation, see [DH84, VIII, Théorème 1]. For $v>0$ the equipotential $v$ of $\mathcal{M}$ is by definition

$$
\mathcal{E}(v):=\Phi^{-1}(\{z \in \mathbb{C}|| z \mid=v\}) .
$$

On the other hand, for $t$ in $\mathbb{R} / \mathbb{Z}$ the set

$$
\mathcal{R}(t):=\Phi^{-1}(\{r \exp (2 \pi i t) \mid r>1\})
$$

is called the external ray of angle $t$ of $\mathcal{M}$. We say that $\mathcal{R}(t)$ lands at a point $z$ in $\mathbb{C}$, if $\Phi^{-1}(r \exp (2 \pi i t))$ converges to $z$ as $r \searrow 1$. When this happens $z$ belongs to $\partial \mathcal{M}$. 
2.3. The wake $1 / 2$. In this subsection we recall a few facts that can be found for example in [DH84] or Mil00].

The external rays $\mathcal{R}(1 / 3)$ and $\mathcal{R}(2 / 3)$ of $\mathcal{M}$ land at the parameter $c=-3 / 4$, and these are the only external rays of $\mathcal{M}$ that land at this point, see for example Mil00, Theorem 1.2]. In particular, the complement in $\mathbb{C}$ of the set

$$
\mathcal{R}(1 / 3) \cup \mathcal{R}(2 / 3) \cup\{-3 / 4\}
$$

has 2 connected components; we denote by $\mathcal{W}$ the connected component containing the point $c=-2$ of $\mathcal{M}$.

For each parameter $c$ in $\mathcal{W}$ the map $f_{c}$ has 2 distinct fixed points; one of the them is the landing point of the external ray $R_{c}(0)$ and it is denoted by $\beta(c)$; the other one is denoted by $\alpha(c)$. The only external ray landing at $\beta(c)$ is $R_{c}(0)$, and the only external ray landing at $-\beta(c)$ is $R_{c}(1 / 2)$.

Moreover, for every parameter $c$ in $\mathcal{W}$ the only external rays of $f_{c}$ landing at $\alpha(c)$ are $R_{c}(1 / 3)$ and $R_{c}(2 / 3)$, see for example [Mil00, Theorem 1.2]. The complement of $R_{c}(1 / 3) \cup R_{c}(2 / 3) \cup\{\alpha(c)\}$ in $\mathbb{C}$ has 2 connected components; one containing $-\beta(c)$ and $z=c$, and the other one containing $\beta(c)$ and $z=0$. On the other hand, the point $\alpha(c)$ has 2 preimages by $f_{c}$ : Itself and $\widetilde{\alpha}(c):=-\alpha(c)$. The only external rays landing at $\widetilde{\alpha}(c)$ are $R_{c}(1 / 6)$ and $R_{c}(5 / 6)$.

2.4. Yoccoz puzzles and para-puzzle. In this subsection we recall the definitions of Yoccoz puzzle and para-puzzle. We follow [Roe00].

Definition 2.1 (Yoccoz puzzles). Fix $c$ in $\mathcal{W}$ and consider the open region $X_{c}:=$ $\left\{z \in \mathbb{C} \mid G_{c}(z)<1\right\}$. The Yoccoz puzzle of $f_{c}$ is given by the following sequence of graphs $\left(I_{c, n}\right)_{n=0}^{+\infty}$ defined for $n=0$ by:

$$
I_{c, 0}:=\partial X_{c} \cup\left(X_{c} \cap \operatorname{cl}\left(R_{c}(1 / 3)\right) \cap \operatorname{cl}\left(R_{c}(2 / 3)\right)\right),
$$

and for $n \geq 1$ by $I_{c, n}:=f_{c}^{-n}\left(I_{c, 0}\right)$. The puzzle pieces of depth $n$ are the connected components of $f_{c}^{-n}\left(X_{c}\right) \backslash I_{c, n}$. The puzzle piece of depth $n$ containing a point $z$ is denoted by $P_{c, n}(z)$.

Note that for a real parameter $c$, every puzzle piece intersecting the real line is invariant under complex conjugation. Since puzzle pieces are simply-connected, it follows that the intersection of such a puzzle piece with $\mathbb{R}$ is an interval.

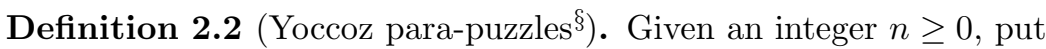

$$
J_{n}:=\left\{t \in[1 / 3,2 / 3] \mid 2^{n} t(\bmod 1) \in\{1 / 3,2 / 3\}\right\},
$$

let $\mathcal{X}_{n}$ be the intersection of $\mathcal{W}$ with the open region in the parameter plane bounded by the equipotential $\mathcal{E}\left(2^{-n}\right)$ of $\mathcal{M}$, and put

$$
\mathcal{I}_{n}:=\partial \mathcal{X}_{n} \cup\left(\mathcal{X}_{n} \cap \bigcup_{t \in J_{n}} \operatorname{cl}(\mathcal{R}(t))\right) .
$$

Then the Yoccoz para-puzzle of $\mathcal{W}$ is the sequence of graphs $\left(\mathcal{I}_{n}\right)_{n=0}^{+\infty}$. The parapuzzle pieces of depth $n$ are the connected components of $\mathcal{X}_{n} \backslash \mathcal{I}_{n}$. The para-puzzle piece of depth $n$ containing a parameter $c$ is denoted by $\mathcal{P}_{n}(c)$.

\footnotetext{
${ }^{\S}$ In contrast with [Roe00], we only consider para-puzzles contained in $\mathcal{W}$.
} 
Observe that there is only 1 para-puzzle piece of depth 0 , and only 1 parapuzzle piece of depth 1; they are bounded by the same external rays but different equipotentials. Both of them contain $c=-2$.

Fix a parameter $c$ in $\mathcal{P}_{0}(-2)$. There are precisely 2 puzzle pieces of depth 0 : $P_{c, 0}(\beta(c))$ and $P_{c, 0}(-\beta(c))$. Each of them is bounded by the equipotential 1 and by the closures of the external rays landing at $\alpha(c)$. Furthermore, the critical value $c$ of $f_{c}$ is contained in $P_{c, 0}(-\beta(c))$ and the critical point in $P_{c, 0}(\beta(c))$. It follows that the set $f_{c}^{-1}\left(P_{c, 0}(\beta(c))\right)$ is the disjoint union of $P_{c, 1}(-\beta(c))$ and $P_{c, 1}(\beta(c))$, so $f_{c}$ maps each of the sets $P_{c, 1}(-\beta(c))$ and $P_{c, 1}(\beta(c))$ biholomorphically to $P_{c, 0}(\beta(c))$. Moreover, there are precisely 3 puzzle pieces of depth 1 :

$$
P_{c, 1}(-\beta(c)), P_{c, 1}(0) \text { and } P_{c, 1}(\beta(c)) ;
$$

$P_{c, 1}(-\beta(c))$ is bounded by the equipotential $1 / 2$ and by the closures of the external rays that land at $\alpha(c) ; P_{c, 1}(\beta(c))$ is bounded by the equipotential $1 / 2$ and by the closures of the external rays that land at $\widetilde{\alpha}(c)$; and $P_{c, 1}(0)$ is bounded by the equipotential $1 / 2$ and by the closures of the external rays that land at $\alpha(c)$ and at $\widetilde{\alpha}(c)$. In particular, the closure of $P_{c, 1}(\beta(c))$ is contained in $P_{c, 0}(\beta(c))$. It follows from this that for each integer $n \geq 1$ the map $f_{c}^{n}$ maps $P_{c, n}(-\beta(c))$ biholomorphically to $P_{c, 0}(\beta(c))$.

The following is used several times, see [CRL12, Lemma 3.3].

Lemma 2.3. For each integer $n \geq 1$, the following properties hold.

1. The para-puzzle piece $\mathcal{P}_{n}(-2)$ contains the closure of $\mathcal{P}_{n+1}(-2)$.

2. For each parameter $c$ in $\mathcal{P}_{n}(-2)$ the critical value $c$ of $f_{c}$ is in $P_{c, n}(-\beta(c))$.

2.5. The uniformly expanding Cantor set. For a parameter $c$ in $\mathcal{P}_{3}(-2)$, the maximal invariant set $\Lambda_{c}$ of $f_{c}^{3}$ in $P_{c, 1}(0)$ plays an important role in the proof of the Main Theorem. After recalling some of the properties of $\Lambda_{c}$ shown in CRL12, $\S 3.3]$, in this subsection we prove that $f_{c}^{3}$ is uniformly expanding on $\Lambda_{c}$ and make some distortion estimates for $f_{c}^{3}$ on $\Lambda_{c}$ (Lemma 2.4).

Fix $c$ in $\mathcal{P}_{3}(-2)$. There are precisely 2 connected components of $f_{c}^{-3}\left(P_{c, 1}(0)\right)$ contained in $P_{c, 1}(0)$ that we denote by $Y_{c}$ and $\tilde{Y}_{c}$. The closures of these sets are disjoint and contained in $P_{c, 1}(0)$. The sets $Y_{c}$ and $\tilde{Y}_{c}$ are distinguished by the fact that $Y_{c}$ contains in its boundary the common landing point of the external rays $R_{c}(7 / 24)$ and $R_{c}(17 / 24)$, denoted $\omega(c)$, and that $\tilde{Y}_{c}$ contains in its boundary the common landing point of the external rays $R_{c}(5 / 24)$ and $R_{c}(19 / 24)$. The map $f_{c}^{3}$ maps each of the sets $Y_{c}$ and $\widetilde{Y}_{c}$ biholomorphically to $P_{c, 1}(0)$. Thus, if we put

then

$$
\begin{aligned}
g_{c}: Y_{c} \cup \tilde{Y}_{c} & \rightarrow P_{c, 1}(0) \\
z & \mapsto g_{c}(z):=f_{c}^{3}(z),
\end{aligned}
$$

$$
\Lambda_{c}=\bigcap_{n \in \mathbb{N}} g_{c}^{-n}\left(\operatorname{cl}\left(P_{c, 1}(0)\right)\right)
$$

The rest of this section is dedicated to prove the following lemma.

Lemma 2.4. There are constants $C_{0}>0$ and $v_{0}>0$ such that for every parameter $c$ in $\mathcal{P}_{5}(-2)$, every $\ell$ in $\mathbb{N}$, and every connected component $W$ of $g_{c}^{-\ell}\left(P_{c, 1}(0)\right)$, we have

$$
\operatorname{diam}(W) \leq C_{0} \exp \left(-v_{0} \ell\right)
$$


furthermore, for all $z$ and $w$ in $W$ we have

$$
\left|\frac{D g_{c}(z)}{D g_{c}(w)}-1\right| \leq C_{0} \exp \left(-v_{0} \ell\right) \text { and } \log \left|\frac{D g_{c}(z)}{D g_{c}(w)}\right| \leq C_{0} \exp \left(-v_{0} \ell\right) \text {. }
$$

To prove this lemma, we recall some facts from [CRL12, §4.1]. For a parameter $c$ in $\mathcal{P}_{2}(-2)$, the open disk $\widehat{U}_{c}$ containing $-\beta(c)$ that is bounded by the equipotential 2 and by

$$
R_{c}(7 / 24) \cup\{\omega(c)\} \cup R_{c}(17 / 24),
$$

contains the closure of $P_{c, 0}(-\beta(c))$ and is disjoint from $P_{c, 1}(\beta(c))$; the set $\widehat{W}_{c}:=$ $f_{c}^{-1}\left(\widehat{U}_{c}\right)$ contains the closure of $P_{c, 1}(0)$ and depends continuously with $c$ on $\mathcal{P}_{3}(-2)$.

Lemma 2.5. For every parameter $c$ in $\mathcal{P}_{4}(-2)$, each of the maps

$$
\psi_{c}:=\left(\left.g_{c}\right|_{Y_{c}}\right)^{-1} \text { and } \widetilde{\psi}_{c}:=\left(\left.g_{c}\right|_{\widetilde{Y}_{c}}\right)^{-1}
$$

extends biholomorphically to $\widehat{W}_{c}$. Moreover, the closures of $\psi_{c}\left(\widehat{W}_{c}\right)$ and $\widetilde{\psi}_{c}\left(\widehat{W}_{c}\right)$ are both included in $P_{c, 1}(0)$.

Proof. Fix a parameter $c$ in $\mathcal{P}_{4}(-2)$.

To prove the first assertion, it is sufficient to show that for $j$ in $\{0,1,2\}$ the critical value $c$ is not in $f_{c}^{-j}\left(\widehat{W}_{c}\right)$. By part 2 of Lemma 2.3, the critical value $c$ is in $P_{c, 4}(-\beta(c))$. Then for $i$ in $\{1,2,3\}$ the point $f_{c}^{i}(c)$ is in the set $P_{c, 1}(\beta(c))$ that is disjoint from $\widehat{U}_{c}$. Using $\widehat{W}_{c}=f_{c}^{-1}\left(\widehat{U}_{c}\right)$, we conclude the proof of the extension.

To prove the second assertion, we use the fact that $f_{c}\left(Y_{c}\right)=f_{c}\left(\widetilde{Y}_{c}\right)$ and that $f_{c}^{2}\left(Y_{c}\right)$ is contained in $P_{c, 1}(\beta(c))$ ( $c f$. ., proof of [CRL12, Lemma 3.5]). Denote by $\widetilde{U}_{c}$ the open disk containing 0 that is bounded by the equipotential 2 , the point $\widetilde{\alpha}(c)$ and the external rays landing at $\widetilde{\alpha}(c)$. Observe that $\widehat{U}_{c} \subset \widetilde{U}_{c}$ and thus, that $\widehat{W}_{c}$ is contained in the connected set $f_{c}^{-1}\left(\widetilde{U}_{c}\right)$. The set $f_{c}^{-1}\left(\widetilde{U}_{c}\right)$ is contained in the set containing $\beta(c)$ and that is bounded by the equipotential 1 , by the preimage $\alpha_{1}(c)$ of $\widetilde{\alpha}(c)$ contained in $P_{c, 1}(-\beta(c))$, and by the external rays $R_{c}(5 / 12)$ and $R_{c}(7 / 12)$ that land at $\alpha_{1}(c)$. In particular, $f_{c}^{-1}\left(\widetilde{U}_{c}\right)$ is disjoint from $P_{c, 4}(-\beta(c))$. This implies that $f_{c}^{-2}\left(\widetilde{U}_{c}\right)$ has 2 connected components, one that is disjoint from $P_{c, 1}(\beta(c))$ and the other one that contains $f_{c}^{2}\left(Y_{c}\right)$; the closure of the latter is contained in $P_{c, 0}(\beta(c))$. Since $f_{c}^{2}\left(P_{c, 1}(0)\right)$ contains $P_{c, 0}(\beta(c))$, we conclude that the closures of the connected components of $f_{c}^{-4}\left(\widetilde{U}_{c}\right)$ containing $Y_{c}$ and $\widetilde{Y}_{c}$ are both contained in $P_{c, 1}(0)$. This proves that the closures of $\psi_{c}\left(\widehat{W}_{c}\right)$ and $\widetilde{\psi}_{c}\left(\widehat{W}_{c}\right)$ are both contained in $P_{c, 1}(0)$.

Proof of Lemma 2.4. By part 1 of Lemma 2.3, the closure of $\mathcal{P}_{5}(-2)$ is a compact set included in $\mathcal{P}_{4}(-2)$. Since $P_{c, 1}(0)$ and $\widehat{W}_{c}$ vary continuously with $c$ in $\mathcal{P}_{4}(-2)$ ( $c f$. , CRL12, Lemma 2.5]), the same holds for

$$
W_{c}:=\psi_{c}\left(\widehat{W}_{c}\right) \text { and } \widetilde{W}_{c}:=\widetilde{\psi}_{c}\left(\widehat{W}_{c}\right) .
$$

Therefore, by Lemma 2.5 we have

$$
\begin{aligned}
A:=\inf _{c \in \mathcal{P}_{5}(-2)} \min \left\{\bmod \left(\widehat{W}_{c} \backslash \operatorname{cl}\left(W_{c}\right)\right), \bmod \left(\widehat{W}_{c} \backslash \operatorname{cl}\left(\widetilde{W}_{c}\right)\right)\right\}>0, \\
\Xi_{0}:=\inf _{c \in \mathcal{P}_{5}(-2)} \operatorname{dist}\left(\partial \widehat{W}_{c}, P_{c, 1}(0)\right)>0, \\
\Xi_{1}:=\sup _{c \in \mathcal{P}_{5}(-2)} \operatorname{diam}\left(P_{c, 1}(0)\right)<+\infty
\end{aligned}
$$


and

$$
\Xi_{2}:=\sup _{c \in \mathcal{P}_{5}(-2)} \sup _{z \in \mathbb{C},|z| \leq 2 \Xi_{1}}\left|D f_{c}^{3}(z)\right|<+\infty .
$$

For an open topological disk $U$ in $\mathbb{C}$, denote by $\operatorname{dist}_{U}$ the Poincaré distance on $U$. Note that there is a constant $\widehat{C}>0$ that only depends on $\Xi_{0}$, such that for every $c$ in $\mathcal{P}_{5}(-2)$ the Euclidean and Poincaré distances on $\widehat{W}_{c}$ are comparable by a factor of $\widehat{C}$ on $P_{c, 1}(0)$, see for example [Mil06, Lemma A.8]. On the other hand, by Pick's Theorem (see for instance [Mil06]), for every parameter $c$ in $\mathcal{P}_{4}(-2)$ the maps $\psi_{c}$ and $\widetilde{\psi}_{c}$ are isometries for the Poincaré distances on $\widehat{W}_{c}$ and on $W_{c}$ and $\widetilde{W}_{c}$, respectively. Again by Pick's Theorem, each of the inclusion maps from $W_{c}$ and $\widetilde{W}_{c}$ into $\widehat{W}_{c}$ are contractions for the corresponding Poincaré distances. It follows that there is $v_{0}>0$ that only depends on $A$, such that each of these inclusions contracts by a factor at least $\exp \left(-v_{0}\right)$. Thus, for every parameter $c$ in $\mathcal{P}_{5}(-2)$ and all $x$ and $y$ in $\widehat{W}_{c}$, we have

$$
\operatorname{dist}_{\widehat{W}_{c}}\left(\psi_{c}(x), \psi_{c}(y)\right) \leq \exp \left(-v_{0}\right) \operatorname{dist}_{\widehat{W}_{c}}(x, y)
$$

and

$$
\operatorname{dist}_{\widehat{W}_{c}}\left(\widetilde{\psi}_{c}(x), \widetilde{\psi}_{c}(y)\right) \leq \exp \left(-v_{0}\right) \operatorname{dist}_{\widehat{W}_{c}}(x, y) .
$$

Let $\ell \geq 1$ be an integer and $W$ a connected component of $g_{c}^{-\ell}\left(P_{c, 1}(0)\right)$. Note that $\left(\left.g_{c}^{\ell}\right|_{W}\right)^{-1}$ extends to a holomorphic map $\psi$ defined on $\widehat{W}_{c}$ that can be written as the composition of $\ell$ maps in $\left\{\psi_{c}, \widetilde{\psi}_{c}\right\}$. Thus,

$$
\operatorname{diam}(W)=\operatorname{diam}\left(\psi\left(P_{c, 1}(0)\right)\right) \leq \widehat{C}^{2} \exp \left(-v_{0} \ell\right) \operatorname{diam}\left(P_{c, 1}(0)\right) .
$$

This proves the first desired estimate with $C_{0}=\widehat{C}^{2} \Xi_{1}$.

To prove the remaining estimates, note that for each point $w$ in $Y_{c} \cup \widetilde{Y}_{c}$ and every $z$ in $\mathbb{C}$ satisfying $|z|=2 \Xi_{1}$, we have

$$
|z-w| \geq \Xi_{1} \text { and }\left|D f_{c}^{3}(z)-D f_{c}^{3}(w)\right| \leq 2 \Xi_{2} .
$$

So for each $w$ in $Y_{c} \cup \widetilde{Y}_{c}$ the maximum principle applied to the holomorphic function

$$
z \mapsto \frac{D f_{c}^{3}(z)-D f_{c}^{3}(w)}{z-w}
$$

and to $\left\{z \in \mathbb{C}|| z \mid \leq 2 \Xi_{1}\right\}$, gives for every $z$ in $Y_{c} \cup \widetilde{Y}_{c}$

$$
\left|D g_{c}(z)-D g_{c}(w)\right|=\left|D f_{c}^{3}(z)-D f_{c}^{3}(w)\right| \leq 2 \Xi_{2} \Xi_{1}^{-1}|z-w| .
$$

On the other hand, since each of the maps $\psi_{c}$ and $\widetilde{\psi}_{c}$ is a contraction for the Poincaré distance on $\widehat{W}_{c}$, by the definition of $\widehat{C}$ we have for every $w$ in $Y_{c} \cup \widetilde{Y}_{c}$ that $\left|D g_{c}(w)\right|^{-1} \leq \widehat{C}^{2}$. We conclude that for all $z$ and $w$ in $Y_{c}$ or in $\widetilde{Y}_{c}$, we have

$$
\left|\frac{D g_{c}(z)}{D g_{c}(w)}-1\right| \leq 2 \widehat{C}^{2} \Xi_{2} \Xi_{1}^{-1}|z-w| \text {. }
$$

Together with the first estimate of the lemma, this implies the second and third estimates with $C_{0}=\left(2 \widehat{C}^{2} \Xi_{2} \Xi_{1}^{-1}\right)\left(\widehat{C}^{2} \Xi_{1}\right)$. 
2.6. Parameters. The parameter we use to prove the Main Theorem is chosen from a set introduced in [CRL12, Proposition 3.1]. In this subsection we recall the definition of this parameter set, and give some dynamical properties of the corresponding maps.

Given an integer $n \geq 3$, let $\mathcal{K}_{n}$ be the set of all those real parameters $c$ such that the following properties hold:

1. We have $c<0$, and for each $j$ in $\{1, \ldots, n-1\}$ we have $f_{c}^{j}(c)>0$.

2. For every integer $k \geq 0$, we have

$$
f_{c}^{n+3 k+1}(c)<0 \text { and } f_{c}^{n+3 k+2}(c)>0 .
$$

Note that for a parameter $c$ in $\mathcal{K}_{n}$ the critical point of $f_{c}$ cannot be asymptotic to a periodic point, see [MT88, §8]. This implies that all the periodic points of $f_{c}$ in $\mathbb{C}$ are hyperbolic repelling and therefore that $K_{c}=J_{c}$, see Mil06. On the other hand, we have $f_{c}(c)>c$ and the interval $I_{c}=\left[c, f_{c}(c)\right]$ is invariant by $f_{c}$. This implies that $I_{c}$ is contained in $J_{c}$ and hence that for every real number $t$ we have $P_{c}^{\mathbb{R}}(t) \leq P_{c}^{\mathbb{C}}(t)$. Note also that $\left.f_{c}\right|_{I_{c}}$ is not renormalizable, so $f_{c}$ is topologically exact on $I_{c}$, see for example [MvS93, Thoerem III.4.1].

Since for $c$ in $\mathcal{K}_{n}$ the critical point of $f_{c}$ is not periodic, for every integer $k \geq 0$ we have $f_{c}^{n+3 k}(c) \neq 0$. Thus, we can define the sequence $\iota(c)$ in $\{0,1\}^{\mathbb{N}_{0}}$ for each $k \geq 0$ by

$$
\iota(c)_{k}:= \begin{cases}0 & \text { if } f_{c}^{n+3 k}(c)<0 \\ 1 & \text { if } f_{c}^{n+3 k}(c)>0 .\end{cases}
$$

Proposition 2.6. For each integer $n \geq 3$, the set $\mathcal{K}_{n}$ is a compact subset of

$$
\mathcal{P}_{n}(-2) \cap(-2,-3 / 4),
$$

and for every sequence $\underline{x}$ in $\{0,1\}^{\mathbb{N}_{0}}$ there is a unique parameter $c$ in $\mathcal{K}_{n}$ such that $\iota(c)=\underline{x}$. Finally, for each $\delta>0$ there is $n_{0} \geq 3$ such that for each integer $n \geq$ $n_{0}$ the set $\mathcal{K}_{n}$ is contained in the interval $(-2,-2+\delta)$.

Recall that for an open subset $G$ of $\mathbb{C}$ and a univalent map $f: G \rightarrow \mathbb{C}$, the distortion of $f$ on a subset $C$ of $G$ is by definition

$$
\sup _{x, y \in C}|D f(x)| /|D f(y)| \text {. }
$$

The following is a uniform distortion bound for parameters as in the previous proposition.

Lemma 2.7 (CRL12, Lemma 4.3). There is a constant $\Delta_{0}>1$ such that for each integer $n \geq 4$ and each parameter $c$ in $\mathcal{K}_{n}$ the following properties hold: For each integer $m \geq 1$ and each connected component $W$ of $f_{c}^{-m}\left(P_{c, 1}(0)\right)$ on which $f_{c}^{m}$ is univalent, $f_{c}^{m}$ maps a neighborhood of $W$ biholomorphically to $\widehat{W}_{c}$ and the distortion of this map on $W$ is bounded by $\Delta_{0}$.

2.7. Induced map and pressure function. Let $n \geq 5$ be an integer and $c$ a parameter in $\mathcal{K}_{n}$. Throughout the rest of this subsection we put $\widehat{V}_{c}:=P_{c, 4}(0)$. Note that the critical value $c$ of $f_{c}$ is in $P_{c, n}(-\beta(c)$ ) (part 2 of Lemma 2.3 and Proposition 2.6), so the closure of

$$
V_{c}:=P_{c, n+1}(0)=f_{c}^{-1}\left(P_{c, n}(-\beta(c))\right)
$$

is contained in $\widehat{V}_{c}=f_{c}^{-1}\left(P_{c, 3}(-\beta(c))\right)$, cf. [CRL12, part 1 of Lemma 3.2]. 
Let $D_{c}$ be the set of all those points $z$ in $V_{c}$ for which there is an integer $m \geq 1$ such that $f_{c}^{m}(z)$ is in $V_{c}$. For $z$ in $D_{c}$ we denote by $m_{c}(z)$ the least integer $m$ with this property, and call it the first return time of $z$ to $V_{c}$. The first return map to $V_{c}$ is defined by

$$
\begin{aligned}
F_{c}: D_{c} & \rightarrow V_{c} \\
z & \mapsto F_{c}(z):=f_{c}^{m_{c}(z)}(z) .
\end{aligned}
$$

It is easy to see that $D_{c}$ is a disjoint union of puzzle pieces; so each connected component of $D_{c}$ is a puzzle piece. Note furthermore that in each of these puzzle pieces $W$, the return time function $m_{c}$ is constant; denote the common value of $m_{c}$ on $W$ by $m_{c}(W)$.

Denote by $\mathfrak{D}_{c}$ the collection of connected components of $D_{c}$ and by $\mathfrak{D}_{c}^{\mathbb{R}}$ the subcollection of $\mathfrak{D}_{c}$ of those sets intersecting $\mathbb{R}$. For each $W$ in $\mathfrak{D}_{c}$ denote by $\phi_{W}: \widehat{V}_{c} \rightarrow$ $V_{c}$ the extension of $\left.F_{c}\right|_{W} ^{-1}$ given by [CRL12, Lemma 6.1]. Given an integer $\ell \geq 1$ we denote by $E_{c, \ell}\left(\right.$ resp. $\left.E_{c, \ell}^{\mathbb{R}}\right)$ the set of all words of length $\ell$ in the alphabet $\mathfrak{D}_{c}$ (resp. $\left.\mathfrak{D}_{c}^{\mathbb{R}}\right)$. Again by [CRL12, Lemma 6.1], for each integer $\ell \geq 1$ and each word $W_{1} \cdots W_{\ell}$ in $E_{c, \ell}$ the composition

$$
\phi_{W_{1} \cdots W_{\ell}}=\phi_{W_{1}} \circ \cdots \circ \phi_{W_{\ell}}
$$

is defined on $\widehat{V}_{c}$. We also put

$$
m_{c}\left(W_{1} \cdots W_{\ell}\right)=m_{c}\left(W_{1}\right)+\cdots+m_{c}\left(W_{\ell}\right) .
$$

For $t, p$ in $\mathbb{R}$ and an integer $\ell \geq 1$ put

$$
Z_{\ell}(t, p):=\sum_{\underline{W} \in E_{c, \ell}} \exp \left(-m_{c}(\underline{W}) p\right)\left(\sup \left\{\left|D \phi_{\underline{W}}(z)\right| \mid z \in V_{c}\right\}\right)^{t}
$$

and

$$
Z_{\ell}^{\mathbb{R}}(t, p):=\sum_{\underline{W} \in E_{c, \ell}^{\mathbb{R}}} \exp \left(-m_{c}(\underline{W}) p\right)\left(\sup \left\{\left|D \phi_{\underline{W}}(z)\right| \mid z \in V_{c}\right\}\right)^{t} .
$$

For a fixed $t$ and $p$ in $\mathbb{R}$ the sequence

$$
\left(\frac{1}{\ell} \log Z_{\ell}(t, p)\right)_{\ell=1}^{+\infty}\left(\operatorname{resp} .\left(\frac{1}{\ell} \log Z_{\ell}^{\mathbb{R}}(t, p)\right)_{\ell=1}^{+\infty}\right)
$$

converges to the pressure function of $F_{c}\left(\right.$ resp. $\left.\left.F_{c}\right|_{D_{c} \cap \mathbb{R}}\right)$ for the potential $-t \log \left|D F_{c}\right|-$ $p m_{c}$; we denote it by $\mathscr{P}_{c}^{\mathbb{C}}(t, p)$ (resp. $\left.\mathscr{P}_{c}^{\mathbb{R}}(t, p)\right)$. On the set where it is finite, the function $\mathscr{P}_{c}^{\mathbb{C}}$ (resp. $\mathscr{P}_{c}^{\mathbb{R}}$ ) so defined is strictly decreasing in each of its variables.

\section{THE 2 VARIABLES SERIES}

We start this section stating a stronger version of the Main Theorem in 3.1 The rest of this section is dedicated to estimate, for a real parameter $c$ in $\bigcup_{n=6}^{+\infty} \mathcal{K}_{n}$ satisfying some mild hypotheses, a certain "postcritical series" in terms of an abstract 2 variables series (Proposition $\mathrm{A}$ in $\$ 3.3$ ). The postcritical series is used in $\$ 5$ to estimate the geometric pressure function. The definition of the 2 variables series is based on an approximation of the derivatives $\left(D f_{c}^{n}(c)\right)_{n=1}^{+\infty}$, using the derivatives of $g_{c}$ at its fixed points $p(c)$ and $\widetilde{p}(c)$. This approximation, which is more precise than a direct application of the Koebe principle, incorporates an estimate of the corresponding distortion constants (Proposition 3.1 in 3.2). This estimate is given in terms of the total distortion of the 2 homoclinic orbits of $g_{c}$ connecting $p(c)$ and $\widetilde{p}(c)$. 
3.1. Main Technical Theorem. In this subsection we state the Main Technical Theorem from which the Main Theorem follows directly. The rest of the paper is dedicated to the proof of the Main Technical Theorem.

Let $c$ be a parameter in $\bigcup_{n=6}^{+\infty} \mathcal{K}_{n}$. An invariant probability measure supported on $I_{c}$ (resp. $J_{c}$ ) is said to be an equilibrium state of $\left.f_{c}\right|_{I_{c}}$ (resp. $f_{c}$ ) for the potential $-\log \left|D f_{c}\right|$, if the supremum defining $P_{c}^{\mathbb{R}}(t)$ (resp. $P_{c}^{\mathbb{C}}(t)$ ) is attained at this measure. Given $t>0$ and a real number $p$ we say a measure $\mu$ is $(t, p)$-conformal for $\left.f_{c}\right|_{I_{c}}$ (resp. $f_{c}$ ), if for every subset $U$ of $I_{c}$ (resp. $J_{c}$ ) on which $\left.f_{c}\right|_{I_{c}}\left(\operatorname{resp} . f_{c}\right)$ is injective we have

$$
\begin{aligned}
\mu\left(\left.f_{c}\right|_{I_{c}}(U)\right)=\exp (p) \int_{U}\left|D f_{c}\right|^{t} d \mu & \left(\text { resp. } \mu\left(f_{c}(U)\right)=\exp (p) \int_{U}\left|D f_{c}\right|^{t} d \mu\right) .
\end{aligned}
$$

In the case where $P_{c}^{\mathbb{R}}(t)=0$ (resp. $P_{c}^{\mathbb{C}}(t)=0$ ), a $(t, 0)$-conformal measure is simply called conformal.

For each $c$ in $\mathcal{P}_{3}(-2)$ denote by $p(c)$ the unique fixed point of $g_{c}$ in $Y_{c}$ and by $\widetilde{p}(c)$ the unique fixed point of $g_{c}$ in $\tilde{Y}_{c}$. Each of the functions

$$
p: \mathcal{P}_{3}(-2) \rightarrow \mathbb{C} \text { and } \tilde{p}: \mathcal{P}_{3}(-2) \rightarrow \mathbb{C}
$$

so defined is holomorphic.

Main Technical Theorem. There is $n_{1} \geq 6$ such that for every integer $n \geq n_{1}$ there are a parameter $c$ in $\mathcal{K}_{n}$, an integer $q \geq 3$, and real numbers $\kappa$ in $[1,2]$ and $\Delta \geq 1$, such that the following properties are satisfied. Put

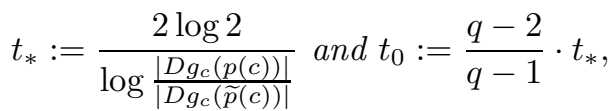

and define the functions $\delta^{+}, \delta^{-}, p^{+}, p^{-}:\left(t_{0},+\infty\right) \rightarrow \mathbb{R}$, by

$$
\begin{gathered}
\delta^{+}(t):= \begin{cases}\frac{2 \log 2}{3} \cdot 2^{-q\left(\frac{\kappa t_{*}}{q\left(t_{*}-t\right)}-1\right)^{2}} & \text { if } t \in\left(t_{0}, t_{*}\right) ; \\
0 & \text { if } t \geq t_{*} ;\end{cases} \\
\delta^{-}(t):= \begin{cases}\frac{\log 2}{3} \cdot 2^{-q\left(\frac{\kappa t_{*}}{q\left(t_{*}-t\right)}+\Delta\right)^{2}} & \text { if } t \in\left(t_{0}, t_{*}\right) ; \\
0 & \text { if } t \geq t_{*} ;\end{cases} \\
p^{+}(t):=-t \frac{\chi_{\mathrm{crit}}(c)}{2}+\delta^{+}(t), \text { and } p^{-}(t):=-t \frac{\chi_{\mathrm{crit}}(c)}{2}+\delta^{-}(t) .
\end{gathered}
$$

Then, $\chi_{\text {crit }}(c)>0$, for $t>t_{0}$ we have

$$
p^{-}(t) \leq P_{c}^{\mathbb{R}}(t) \leq P_{c}^{\mathbb{C}}(t) \leq p^{+}(t),
$$

and for $t \geq t_{*}$ there is no equilibrium state of $\left.f_{c}\right|_{I_{c}}$ (resp. $\left.\left.f_{c}\right|_{J_{c}}\right)$ for the potential - $t \log \left|D f_{c}\right|$ and we have

$$
\mathscr{P}_{c}^{\mathbb{R}}\left(t,-t \frac{\chi_{\mathrm{crit}}(c)}{2}\right) \leq \mathscr{P}_{c}^{\mathbb{C}}\left(t,-t \frac{\chi_{\mathrm{crit}}(c)}{2}\right)<0 .
$$

Moreover, for $t \geq t_{*}$ and for $p$ in $\mathbb{R}$ the following properties hold:

1. If $p \geq-t \chi_{\mathrm{crit}}(c) / 2$, then there is a unique $(t, p)$-conformal probability measure for $\left.f_{c}\right|_{I_{c}}\left(\right.$ resp. $\left.f_{c}\right)$ supported on $I_{c}\left(\right.$ resp. $\left.J_{c}\right)$. Moreover, this measure is dissipative, purely atomic, and supported on the backward orbit of $z=0$. 
2. If $p<-t \chi_{\text {crit }}(c) / 2$, then there is no $(t, p)$-conformal probability measure for $\left.f_{c}\right|_{I_{c}}$ (resp. $f_{c}$ ) supported on $I_{c}$ (resp. $\left.J_{c}\right)$.

3.2. Improved distortion estimate. The purpose of this subsection is to prove Proposition 3.1 below. For the statement, define for each $c$ in $\mathcal{P}_{3}(-2)$ the itinerary map

$$
\iota_{c}: \Lambda_{c} \rightarrow\{0,1\}^{\mathbb{N}_{0}}
$$

for $x$ in $\Lambda_{c}$ and $k$ in $\mathbb{N}_{0}$, by

$$
\iota_{c}(x)_{k}:= \begin{cases}0 & \text { if } g_{c}^{k}(x) \in Y_{c} \\ 1 & \text { if } g_{c}^{k}(x) \in \widetilde{Y}_{c} .\end{cases}
$$

We recall from [CRL12, §3.3] that the map $\iota_{c}$ conjugates the action of $g_{c}$ on $\Lambda_{c}$ to the action of the shift map on $\{0,1\}^{\mathbb{N}_{0}}$. Moreover, if $c$ is real, then $\Lambda_{c}$ is contained in $\mathbb{R}$, the sets

$$
Y_{c} \cap \mathbb{R} \text { and } f_{c}\left(Y_{c} \cap \mathbb{R}\right)=f_{c}\left(\tilde{Y}_{c} \cap \mathbb{R}\right)
$$

are both contained in the negative real numbers, and the sets

$$
\widetilde{Y}_{c} \cap \mathbb{R} \text { and } f_{c}^{2}\left(Y_{c} \cap \mathbb{R}\right)=f_{c}^{2}\left(\widetilde{Y}_{c} \cap \mathbb{R}\right)
$$

are both contained in the positive real numbers. It follows that for $c$ in $\mathcal{K}_{n}$ the point $f_{c}^{n}(c)$ is in $\Lambda_{c}$ and the sequence $\iota(c)$ defined in $\$ 2.6$ is equal to $\iota_{c}\left(f_{c}^{n}(c)\right)$. Finally, for each $\underline{x}$ in $\{0,1\}^{\mathbb{N}_{0}}$ define the function

$$
\begin{aligned}
& I_{\underline{x}}: \mathcal{P}_{3}(-2) \rightarrow \mathbb{C} \\
& c \quad \mapsto \quad I_{\underline{x}}(c):=\iota_{c}^{-1}(\underline{x}) \text {. }
\end{aligned}
$$

By a normality argument the function $I_{\underline{x}}$ is holomorphic.

Proposition 3.1 (Improved distortion estimate). There are analytic functions

$$
\zeta: \mathcal{P}_{5}(-2) \rightarrow(0,+\infty) \text { and } \widetilde{\zeta}: \mathcal{P}_{5}(-2) \rightarrow(0,+\infty)
$$

and constants $C_{1}>0$ and $v_{1}>0$, such that for every integer $n \geq 5$ and every parameter $c$ in $\mathcal{K}_{n}$ the following property holds: Let $m$ and $m^{\prime}$ be positive integers and let

$$
\underline{x}=\left(x_{j}\right)_{j=0}^{+\infty}\left(\operatorname{resp} . \underline{\tilde{x}}=\left(\widetilde{x}_{j}\right)_{j=0}^{+\infty}\right)
$$

be a sequence in $\{0,1\}^{\mathbb{N}_{0}}$ such that for $j$ in $\{0, \ldots, m-1\}$ we have $x_{j}=0$ (resp. $\widetilde{x}_{j}=1$ ) and such that for $j$ in $\left\{0, \ldots, m^{\prime}-1\right\}$ we have $x_{m+j}=1$ (resp. $\widetilde{x}_{m+j}=0$ ). Then

and

$$
\left|\log \frac{\left|D g_{c}^{m}\left(I_{\underline{x}}(c)\right)\right|}{\left|D g_{c}(p(c))\right|^{m}}-\log \zeta(c)\right| \leq C_{1} \exp \left(-\min \left\{m, m^{\prime}\right\} v_{1}\right)
$$

$$
\left|\log \frac{\left|D g_{c}^{m}\left(I_{\widetilde{\underline{x}}}(c)\right)\right|}{\left|D g_{c}(\widetilde{p}(c))\right|^{m}}-\log \widetilde{\zeta}(c)\right| \leq C_{1} \exp \left(-\min \left\{m, m^{\prime}\right\} v_{1}\right) .
$$

The proof of this proposition is at the end of this subsection.

For each integer $\ell$ in $\mathbb{N}_{0}$, let

$$
\underline{x}^{\ell}=\left(x_{j}^{\ell}\right)_{j=0}^{+\infty} \text { and } \underline{\widetilde{x}}^{\ell}=\left(\widetilde{x}_{j}^{\ell}\right)_{j=0}^{+\infty}
$$

be the sequences in $\{0,1\}^{\mathbb{N}_{0}}$ defined for each $j$ in $\mathbb{N}_{0}$ by

$$
x_{j}^{\ell}= \begin{cases}0 & \text { if } j \leq \ell-1 ; \\ 1 & \text { if } j \geq \ell\end{cases}
$$


and

$$
\widetilde{x}_{j}^{\ell}= \begin{cases}1 & \text { if } j \leq \ell-1 \\ 0 & \text { if } j \geq \ell\end{cases}
$$

Observe that for every $c$ in $\mathcal{P}_{3}(-2)$ and for every $\ell$ in $\mathbb{N}$, the points $I_{\underline{\underline{x}}}(c)$ and $p(c)$ are in the same connected component of $g_{c}^{-\ell}\left(P_{c, 1}(0)\right)$, and the same holds for $I_{\underline{x}^{\ell}}(c)$ and $\widetilde{p}(c)$. Thus, the following is a direct consequence of Lemma 2.4 .

Corollary 3.2. Let $C_{0}>0$ and $v_{0}>0$ be the constants given by Lemma 2.4. Then, for every parameter $c$ in $\mathcal{P}_{5}(-2)$ and for every $\ell$ in $\mathbb{N}$ we have

$$
\begin{gathered}
\left|\frac{D g_{c}\left(I_{\underline{x}^{\ell}}(c)\right)}{D g_{c}(p(c))}-1\right| \leq C_{0} \exp \left(-v_{0} \ell\right),\left|\frac{D g_{c}\left(I_{\widetilde{x}^{\ell}}(c)\right)}{D g_{c}(\widetilde{p}(c))}-1\right| \leq C_{0} \exp \left(-v_{0} \ell\right), \\
\log \left|\frac{D g_{c}\left(I_{\underline{x}^{\ell}}(c)\right)}{D g_{c}(p(c))}\right| \leq C_{0} \exp \left(-v_{0} \ell\right) \text { and } \log \left|\frac{D g_{c}\left(I_{\widetilde{x}^{\ell}}(c)\right)}{D g_{c}(\widetilde{p}(c))}\right| \leq C_{0} \exp \left(-v_{0} \ell\right) .
\end{gathered}
$$

Lemma 3.3 (Homoclinic distortion). For every parameter $c$ in $\mathcal{P}_{5}(-2)$ the limits

$$
\zeta(c):=\lim _{m \rightarrow+\infty} \prod_{\ell=1}^{m} \frac{\left|D g_{c}\left(I_{\underline{x}^{\ell}}(c)\right)\right|}{\left|D g_{c}(p(c))\right|} \text { and } \widetilde{\zeta}(c):=\lim _{m \rightarrow+\infty} \prod_{\ell=1}^{m} \frac{\left|D g_{c}\left(I_{\widetilde{x}^{\ell}}(c)\right)\right|}{\left|D g_{c}(\widetilde{p}(c))\right|}
$$

exist and depend analytically with $c$ on $\mathcal{P}_{5}(-2)$.

Proof. We prove the existence of the first limit and its analytic dependence on $c$; the proof of the analogous assertions for the second limit are similar.

Denote by $\log$ the logarithm defined in the open disk of $\mathbb{C}$ of radius 1 centered at $z=1$. By Corollary 3.2 there is $\ell_{0}$ in $\mathbb{N}$ such that for every $\ell \geq \ell_{0}$ and every $c$ in $\mathcal{P}_{5}(-2)$ we have $\left|\frac{D g_{c}\left(I_{\underline{\underline{x}}} \ell\right)(c)}{D g_{c}(p(c))}-1\right|<1$, so the logarithm $\log \frac{D g_{c}\left(I_{\underline{\underline{x}}} \ell\right)(c)}{D g_{c}(p(c))}$ is defined. Corollary 3.2 also implies that the sum

$$
\sum_{\ell=\ell_{0}}^{+\infty} \log \frac{D g_{c}\left(I_{\underline{x}^{\ell}}\right)(c)}{D g_{c}(p(c))}
$$

exists and is a holomorphic function of $c$ on $\mathcal{P}_{5}(-2)$. Exponentiating, we obtain that the infinite product $\prod_{\ell=\ell_{0}}^{+\infty} \frac{D g_{c}\left(I_{\underline{x}} \ell\right)(c)}{D g_{c}(p(c))}$ exists and is holomorphic on $\mathcal{P}_{5}(-2)$. This implies that the infinite product starting from $\ell=1$ also exists and is holomorphic on $\mathcal{P}_{5}(-2)$. Taking modulus we conclude the proof.

Proof of Proposition 3.1. Let $C_{0}$ and $v_{0}$ be the constants given by Lemma 2.4 and let

$$
\zeta: \mathcal{P}_{5}(-2) \rightarrow(0,+\infty) \text { and } \widetilde{\zeta}: \mathcal{P}_{5}(-2) \rightarrow(0,+\infty)
$$

be the continuous functions given by Lemma 3.3 . 
We only prove the first inequality, the other inequality being similar. We have

$$
\begin{aligned}
\log \frac{\left|D g_{c}^{m}\left(I_{\underline{x}}(c)\right)\right|}{\left|D g_{c}(p(c))\right|^{m}}-\log \zeta(c) & \\
= & \sum_{j=0}^{m-1} \log \frac{\left|D g_{c}\left(g_{c}^{j}\left(I_{\underline{x}}(c)\right)\right)\right|}{\left|D g_{c}(p(c))\right|}-\lim _{\widetilde{m} \rightarrow+\infty} \sum_{\ell=1}^{\widetilde{m}} \log \frac{\left|D g_{c}\left(I_{\underline{x}^{\ell}}(c)\right)\right|}{\left|D g_{c}(p(c))\right|} \\
= & \sum_{j=0}^{m-1} \log \frac{\left|D g_{c}\left(g_{c}^{j}\left(I_{\underline{x}}(c)\right)\right)\right|}{\left|D g_{c}(p(c))\right|}-\sum_{\ell=1}^{m} \log \frac{\left|D g_{c}\left(I_{\underline{x}^{\ell}}(c)\right)\right|}{\left|D g_{c}(p(c))\right|} \\
& -\lim _{\widetilde{m} \rightarrow+\infty} \sum_{\ell=m+1}^{\widetilde{m}} \log \frac{\left|D g_{c}\left(I_{\underline{x}}^{\ell}(c)\right)\right|}{\left|D g_{c}(p(c))\right|} .
\end{aligned}
$$

Notice that for every $j$ in $\{0, \ldots, m-1\}$ we have $g_{c}^{j}\left(I_{\underline{x}^{m}}(c)\right)=I_{\underline{x}^{m-j}}(c)$, and that the points $g_{c}^{j}\left(I_{\underline{x}}(c)\right)$ and $g_{c}^{j}\left(I_{\underline{x}^{m}}(c)\right)$ are in the same connected component of $g_{c}^{-\left(m+m^{\prime}-j\right)}\left(P_{c, 1}(0)\right)$. Using Lemma 2.4 repeatedly, we get

$$
\begin{gathered}
\left|\sum_{j=0}^{m-1} \log \frac{\left|D g_{c}\left(g_{c}^{j}\left(I_{\underline{x}}(c)\right)\right)\right|}{\left|D g_{c}(p(c))\right|}-\sum_{\ell=1}^{m} \log \frac{\left|D g_{c}\left(I_{\underline{x}^{\ell}}(c)\right)\right|}{\left|D g_{c}(p(c))\right|}\right| \\
=\left|\sum_{j=0}^{m-1} \log \frac{\left|D g_{c}\left(g_{c}^{j}\left(I_{\underline{x}}(c)\right)\right)\right|}{\left|D g_{c}(p(c))\right|}-\sum_{j=0}^{m-1} \log \frac{\left|D g_{c}\left(I_{\underline{x}^{m-j}}(c)\right)\right|}{\left|D g_{c}(p(c))\right|}\right| \\
=\left|\sum_{j=0}^{m-1} \log \frac{\left|D g_{c}\left(g_{c}^{j}\left(I_{\underline{x}}(c)\right)\right)\right|}{\left|D g_{c}\left(I_{\underline{x}^{m-j}}(c)\right)\right|}\right|=\left|\sum_{j=0}^{m-1} \log \frac{\left|D g_{c}\left(g_{c}^{j}\left(I_{\underline{x}}(c)\right)\right)\right|}{\left|D g_{c}\left(g_{c}^{j}\left(I_{\underline{x}^{m}}(c)\right)\right)\right|}\right| \\
\leq C_{0} \sum_{j=0}^{m-1} \exp \left(-v_{0}\left(m+m^{\prime}-j\right)\right) \leq \frac{C_{0} \exp \left(-v_{0}\right)}{1-\exp \left(-v_{0}\right)} \exp \left(-v_{0} m^{\prime}\right) .
\end{gathered}
$$

On the other hand, by Corollary 3.2 we have for every integer $\widetilde{m} \geq m$,

$$
\left|\sum_{\ell=m+1}^{\widetilde{m}} \log \frac{\left|D g_{c}\left(I_{\underline{x}^{\ell}}(c)\right)\right|}{\left|D g_{c}(p(c))\right|}\right| \leq C_{0} \sum_{\ell=m+1}^{\widetilde{m}} \exp \left(-v_{0} \ell\right) \leq \frac{C_{0} \exp \left(-v_{0}\right)}{1-\exp \left(-v_{0}\right)} \exp \left(-v_{0} m\right) \text {. }
$$

Taking $C_{1}:=2 \frac{C_{0} \exp \left(-v_{0}\right)}{1-\exp \left(-v_{0}\right)}$ and $v_{1}:=v_{0}$ we conclude the proof of the proposition.

3.3. The 2 variable series. For each integer $n \geq 4$ and for each parameter $c$ in $\mathcal{K}_{n}$, denote by

$$
\iota(c):=\iota_{c}\left(f_{c}^{n}(c)\right)
$$

the itinerary for $g_{c}$ in the Cantor set $\Lambda_{c}$ of the point $x=f_{c}^{n}(c)$, see 2.6. Furthermore, denote by $N_{c}: \mathbb{N}_{0} \rightarrow \mathbb{N}_{0}$ the function defined by $N_{c}(0):=0$ and for $k$ in $\mathbb{N}$ by

$$
N_{c}(k):=\sharp\left\{j \in\{0, \ldots, k-1\} \mid \iota(c)_{j}=0\right\},
$$

and by $B_{c}: \mathbb{N}_{0} \rightarrow \mathbb{N}_{0}$ the function defined by $B_{c}(0):=0, B_{c}(1):=1$, and for $k \geq 2$ by

$$
B_{c}(k):=1+\sharp\left\{j \in\{0, \ldots, k-2\} \mid \iota(c)_{j} \neq \iota(c)_{j+1}\right\} .
$$


Note that for $k$ in $\mathbb{N}$ the function $B_{c}(k)$ is equal to the number of blocks of 0 's and 1 's in the sequence $\left(\iota(c)_{j}\right)_{j=0}^{k-1}$.

On the other hand, for each parameter $c$ in $\mathcal{P}_{5}(-2)$, define

$$
\theta(c):=\left|\frac{D g_{c}(p(c))}{D g_{c}(\widetilde{p}(c))}\right|^{1 / 2}, \xi(c):=-\frac{\log (\zeta(c) \widetilde{\zeta}(c))}{4 \log \theta(c)}
$$

and the 2 variables series $\Pi_{c}$ on $[0,+\infty) \times[0,+\infty)$ by

$$
\Pi_{c}(\tau, \lambda):=\sum_{k=0}^{+\infty} 2^{-\lambda k-\tau N_{c}(k)+\tau \xi(c) B_{c}(k)} .
$$

The purpose of this subsection is to prove the following proposition.

Proposition A. There are constants $C_{2}>1$ and $v_{2}>0$ such that for every integer $n \geq 6$ the following property holds. Let $c$ be a parameter in $\mathcal{K}_{n}$ such that $N_{c}(k) / k \rightarrow 0$ as $k \rightarrow+\infty$ and such that, if we denote by $\left(m_{j}\right)_{j=0}^{+\infty}$ the sequence of lengths of the blocks of 0 's and 1 's in the sequence $\iota(c)$, then the sum

$$
\sum_{j=0}^{+\infty} \exp \left(-\min \left\{m_{j}, m_{j+1}\right\} v_{2}\right)
$$

converges. Then for all $t>0$ and $\delta \geq 0$, we have

$$
\begin{aligned}
& C_{2}^{-t} \exp (-n \delta)\left(\frac{\exp \left(\chi_{\text {crit }}(c)\right)}{\left|D f_{c}(\beta(c))\right|}\right)^{\frac{t}{2} n} \Pi_{c}\left(t \frac{\log \theta(c)}{\log 2}, \frac{3 \delta}{\log 2}\right) \\
& \leq \sum_{k=0}^{+\infty} \exp \left(-(n+3 k)\left(-t \frac{\chi_{\text {crit }}(c)}{2}+\delta\right)\right)\left|D f_{c}^{n+3 k}(c)\right|^{-\frac{t}{2}} \\
& \quad \leq C_{2}^{t} \exp (-n \delta)\left(\frac{\exp \left(\chi_{\text {crit }}(c)\right)}{\left|D f_{c}(\beta(c))\right|}\right)^{\frac{t}{2} n} \Pi_{c}\left(t \frac{\log \theta(c)}{\log 2}, \frac{3 \delta}{\log 2}\right) .
\end{aligned}
$$

The proof of this proposition is at the end of this subsection.

Lemma 3.4. Let $\Delta_{0}$ be the constant given by Lemma 2.7 and let $C_{1}>0$ and $v_{1}>0$ be the constants given by Proposition [3.1. Moreover, let $n \geq 5$ be an integer, let $c$ be a parameter in $\mathcal{K}_{n}$, and denote by $\left(m_{j}\right)_{j=0}^{+\infty}$ the sequence of lengths of the blocks of 0 's and 1 's in the sequence $\iota(c)$. Then for every integer integer $k$ in $\mathbb{N}$ we have

$$
\begin{aligned}
\Delta_{0}^{-1} \max \left\{\frac{\zeta(c)}{\widetilde{\zeta}(c)}, \frac{\widetilde{\zeta}(c)}{\zeta(c)}\right\}^{-1 / 2} \exp \left(-C_{1} \sum_{j=0}^{B_{c}(k)-1} \exp \left(-\min \left\{m_{j}, m_{j+1}\right\} v_{1}\right)\right) \\
\leq \frac{\left|D g_{c}^{k}\left(f_{c}^{n}(c)\right)\right|}{\left|D g_{c}(\widetilde{p}(c))\right|^{k} \cdot \theta(c)^{2 N_{c}(k)} \cdot(\zeta(c) \widetilde{\zeta}(c))^{B_{c}(k) / 2}} \\
\leq \Delta_{0} \max \left\{\frac{\zeta(c)}{\widetilde{\zeta}(c)}, \frac{\widetilde{\zeta}(c)}{\zeta(c)}\right\}^{1 / 2} \exp \left(C_{1} \sum_{j=0}^{B_{c}(k)-1} \exp \left(-\min \left\{m_{j}, m_{j+1}\right\} v_{1}\right)\right) .
\end{aligned}
$$

Proof. If the first $k$ entries of $\iota(c)$ are equal, then $B_{c}(k)=1$ and the desired assertion follows from Lemma 2.7. Suppose that not all of the first $k$ entries of $\iota(c)$ are equal, and let $k_{0}$ be the maximal element of $\{1, \ldots, k\}$ such that

$$
\iota(c)_{k_{0}-1} \neq \iota(c)_{k_{0}} \text {. }
$$


Moreover, denote by $B$ and $\widetilde{B}$ the number of blocks of 0's and 1's, respectively, in the sequence $\left(\iota(c)_{j}\right)_{j=0}^{k_{0}-1}$. We have $B_{c}\left(k_{0}\right)=B+\widetilde{B}$, and

$$
\left|B_{c}\left(k_{0}\right)-2 B\right|=\left|B_{c}\left(k_{0}\right)-2 \widetilde{B}\right| \leq 1 \text {. }
$$

Consider a block of 0's or 1's in $\iota(c)$ with initial position $i$ and length $m$, and let $m^{\prime}$ be the length of the next block. By Proposition 3.1 we have the following 2 cases: If $\iota(c)_{i}=0$, then

$$
\left|\log \frac{\left|D g_{c}^{m}\left(g_{c}^{i}\left(f_{c}^{n}(c)\right)\right)\right|}{\left|D g_{c}(p(c))\right|^{m}}-\log \zeta(c)\right| \leq C_{1} \exp \left(-\min \left\{m, m^{\prime}\right\} v_{1}\right) ;
$$

and if $\iota(c)_{i}=1$, then

$$
\left|\log \frac{\left|D g_{c}^{m}\left(g_{c}^{i}\left(f_{c}^{n}(c)\right)\right)\right|}{\left|D g_{c}(\widetilde{p}(c))\right|^{m}}-\log \widetilde{\zeta}(c)\right| \leq C_{1} \exp \left(-\min \left\{m, m^{\prime}\right\} v_{1}\right)
$$

Applying these inequalities to each of the blocks of 0's and 1's in $\left(\iota(c)_{j}\right)_{j=0}^{k_{0}-1}$, we obtain

$$
\begin{aligned}
& \exp \left(-C_{1} \sum_{j=0}^{B_{c}\left(k_{0}\right)-1} \exp \left(-\min \left\{m_{j}, m_{j+1}\right\} v_{1}\right)\right) \\
& \leq \frac{\left|D g_{c}^{k_{0}}\left(f_{c}^{n}(c)\right)\right|}{\left|D g_{c}(p(c))\right|^{N_{c}\left(k_{0}\right)}\left|D g_{c}(\widetilde{p}(c))\right|^{k_{0}-N_{c}\left(k_{0}\right)} \zeta(c)^{B} \widetilde{\zeta}(c)^{\widetilde{B}}} \\
& \leq \exp \left(C_{1} \sum_{j=0}^{B_{c}\left(k_{0}\right)-1} \exp \left(-\min \left\{m_{j}, m_{j+1}\right\} v_{1}\right)\right) \text {. }
\end{aligned}
$$

Together with (3.2) this implies the desired chain of inequalities in the case where $k_{0}=$ $k$. If $k_{0} \leq k-1$, then by Lemma 2.7 we have

$$
\Delta_{0}^{-1} \leq \frac{\left|D g_{c}^{k-k_{0}}\left(g_{c}^{k_{0}}\left(f_{c}^{n}(c)\right)\right)\right|}{\left|D g_{c}(\widetilde{p}(c))\right|^{k-k_{0}} \cdot \theta(c)^{2\left(N_{c}(k)-N_{c}\left(k_{0}\right)\right)}} \leq \Delta_{0}
$$

This, together with (3.2), (3.3), and $B_{c}(k)=B_{c}\left(k_{0}\right)+1$, implies the desired chain of inequalities. The proof of the lemma is thus complete.

Lemma 3.5. Let $n \geq 4$ be an integer and let $c$ be a parameter in $\mathcal{K}_{n}$ such that $N_{c}(k) / k \rightarrow 0$ as $k \rightarrow+\infty$. Then we have

$$
\chi_{\text {crit }}(c)=\frac{1}{3} \log \left|D g_{c}(\widetilde{p}(c))\right| .
$$

Proof. Put $\widehat{c}:=f_{c}^{n}(c)$. For every $k$ in $\mathbb{N}$ and every $j$ in $\{0,1,2\}$, we have by the chain rule

$$
\begin{aligned}
D f_{c}^{3 k+j}(c) & =D f_{c}^{j}\left(\left(f_{c}^{3 k}\right)(\widehat{c})\right) \cdot D f_{c}^{3 k}(\widehat{c}) \cdot D f_{c}^{n}(c) \\
& =D f_{c}^{j}\left(g_{c}^{k}(\widehat{c})\right) \cdot D g_{c}^{k}(\widehat{c}) \cdot D f_{c}^{n}(c) .
\end{aligned}
$$

Since $\left|D f_{c}^{j}\left(\left(g_{c}^{k}\right)(\widehat{c})\right)\right|$ is bounded independently of $k$ and $j$, we have

$$
\chi_{\text {crit }}(c)=\liminf _{m \rightarrow+\infty} \frac{1}{m} \log \left|D f_{c}^{m}(c)\right|=\frac{1}{3} \liminf _{k \rightarrow+\infty} \frac{1}{k} \log \left|D g_{c}^{k}(\widehat{c})\right| .
$$


On the other hand, by Lemma 2.7. there is a constant $\Delta_{0}>1$ such that for each integer $k$ in $\mathbb{N}$,

$$
\Delta_{0}^{-B_{c}(k)} \leq \frac{\left|D g_{c}^{k}(\widehat{c})\right|}{\left|D g_{c}(\widetilde{p}(c))\right|^{k-N_{c}(k)}\left|D g_{c}(p(c))\right|^{N_{c}(k)}} \leq \Delta_{0}^{B_{c}(k)}
$$

Taking logarithm yields

$$
\begin{aligned}
-B_{c}(k) \log \Delta_{0}+N_{c}(k) \log \frac{\left|D g_{c}(p(c))\right|}{\left|D g_{c}(\widetilde{p}(c))\right|} & \\
\leq \log \left|D g_{c}^{k}(\widehat{c})\right| & -k \log \left|D g_{c}(\widetilde{p}(c))\right| \\
\leq & B_{c}(k) \log \Delta_{0}+N_{c}(k) \log \frac{\left|D g_{c}(p(c))\right|}{\left|D g_{c}(\widetilde{p}(c))\right|} .
\end{aligned}
$$

Since for each $k$ in $\mathbb{N}$ we have $B_{c}(k) \leq 2 N_{c}(k)+1$, using the hypothesis that $N_{c}(k) / k \rightarrow 0$ as $k \rightarrow+\infty$, we conclude

$$
\lim _{k \rightarrow+\infty} \frac{1}{k} \log \left|D g_{c}^{k}(\widehat{c})\right|=\log \left|D g_{c}(\widetilde{p}(c))\right| .
$$

Combined with (3.4), this completes the proof of the lemma.

Lemma 3.6 (CRL12, Lemma 3.6). There is a constant $\Delta_{1}>1$, such that for each parameter $c$ in $\mathcal{P}_{2}(-2)$, each integer $k \geq 2$, and each point $y$ in $P_{c, k}(-\beta(c))$, we have

$$
\Delta_{1}^{-1}\left|D f_{c}(\beta(c))\right|^{k} \leq\left|D f_{c}^{k}(y)\right| \leq \Delta_{1}\left|D f_{c}(\beta(c))\right|^{k} .
$$

Proof of Proposition $A$. Let $\Delta_{0}$ be the constant given by Lemma 2.7 let $C_{1}$ and $v_{1}$ be the constants given by Proposition 3.1. and let $\Delta_{1}$ be the constant given by Lemma 3.6. Note that by Proposition 3.1 and part 1 of Lemma 2.3.

$$
\Delta:=\sup _{c \in \mathcal{P}_{6}(-2)} \max \left\{\frac{\zeta(c)}{\widetilde{\zeta}(c)}, \frac{\widetilde{\zeta}(c)}{\zeta(c)}\right\}<+\infty .
$$

Let $n, c$, and $\left(m_{j}\right)_{j=0}^{+\infty}$ be as in the statement of the proposition, and put

$$
\sigma:=C_{1} \sum_{j=0}^{+\infty} \exp \left(-\min \left\{m_{j}, m_{j+1}\right\} v_{1}\right) \text { and } \widehat{C}_{2}:=\Delta_{0} \Delta_{1} \Delta^{1 / 2} \exp (\sigma) .
$$

Then for every $k$ in $\mathbb{N}$ and every $t>0$, we have, using

$$
D f_{c}^{n+3 k}(c)=D g_{c}^{k}\left(f_{c}^{n}(c)\right) \cdot D f_{c}^{n}(c)
$$

and combining Lemmas 3.4 and 3.6 .

$$
\begin{aligned}
& \widehat{C}_{2}^{-t} \theta(c)^{-2 t N_{c}(k)}(\zeta(c) \widetilde{\zeta}(c))^{-t B_{c}(k) / 2} \\
& \quad \leq \frac{\left|D f_{c}^{n+3 k}(c)\right|^{-t}}{\left|D g_{c}(\widetilde{p}(c))\right|^{-t k}\left|D f_{c}(\beta(c))\right|^{-t n}} \\
& \quad \leq \widehat{C}_{2}^{t} \theta(c)^{-2 t N_{c}(k)}(\zeta(c) \widetilde{\zeta}(c))^{-t B_{c}(k) / 2}
\end{aligned}
$$

Since by Lemma 3.5 we have

$$
\exp \left((n+3 k) t \chi_{\text {crit }}(c)\right)=\exp \left(n t \chi_{\text {crit }}(c)\right)\left|D g_{c}(\widetilde{p}(c))\right|^{t k},
$$


if we multiply each term in the chain of inequalities (3.5) by

$$
\left(\frac{\exp \left(\chi_{\text {crit }}(c)\right)}{\left|D f_{c}(\beta(c))\right|}\right)^{t n}
$$

then we get

$$
\begin{aligned}
\widehat{C}_{2}^{-t}\left(\frac{\exp \left(\chi_{\text {crit }}(c)\right)}{\left|D f_{c}(\beta(c))\right|}\right)^{t n} & \theta(c)^{-2 t N_{c}(k)}(\zeta(c) \widetilde{\zeta}(c))^{-t B_{c}(k) / 2} \\
\leq & \exp \left((n+3 k) t \chi_{\text {crit }}(c)\right)\left|D f_{c}^{n+3 k}(c)\right|^{-t} \\
& \leq \widehat{C}_{2}^{t}\left(\frac{\exp \left(\chi_{\text {crit }}(c)\right)}{\left|D f_{c}(\beta(c))\right|}\right)^{t n} \theta(c)^{-2 t N_{c}(k)}(\zeta(c) \widetilde{\zeta}(c))^{-t B_{c}(k) / 2} .
\end{aligned}
$$

Taking square roots and then by multiplying by $\exp (-(n+3 k) \delta)$ in each of the terms of the chain of inequalities above, we obtain

$$
\begin{gathered}
\widehat{C}_{2}^{-t / 2} \exp (-n \delta)\left(\frac{\exp \left(\chi_{\text {crit }}(c)\right)}{\left|D f_{c}(\beta(c))\right|}\right)^{\frac{t}{2} n} \exp (-3 k \delta) \theta(c)^{-t N_{c}(k)}(\zeta(c) \widetilde{\zeta}(c))^{-t B_{c}(k) / 4} \\
\leq \exp \left(-(n+3 k)\left(-t \frac{\chi_{\text {crit }}(c)}{2}+\delta\right)\right)\left|D f_{c}^{n+3 k}(c)\right|^{-\frac{t}{2}} \\
\leq \widehat{C}_{2}^{t / 2} \exp (-n \delta)\left(\frac{\exp \left(\chi_{\text {crit }}(c)\right)}{\left|D f_{c}(\beta(c))\right|}\right)^{\frac{t}{2} n} \exp (-3 k \delta) \theta(c)^{-t N_{c}(k)}(\zeta(c) \widetilde{\zeta}(c))^{-t B_{c}(k) / 4} .
\end{gathered}
$$

Note that when $k=0$ this chain of inequalities holds by Lemma 2.7 and our definition of $\widehat{C}_{2}$. Summing over $k \geq 0$, we obtain the proposition with $C_{2}=$ $\widehat{C}_{2}^{1 / 2}$.

\section{Estimating the 2 VARIABles SERIES}

This section is dedicated to estimate, in an abstract setting, the 2 variables series defined in $\$ 3.3$ for a certain itinerary defined in $\$ 4.1$. Our main estimate is stated as Proposition B in 4.2 .

4.1. The itinerary. Given an integer $\Xi$, let $q \geq 3$ be a sufficiently large integer such that $q+\Xi \geq 1$ and $2^{q-1} \geq q+1+\Xi$. Define the quadratic function

$$
\begin{aligned}
Q: \mathbb{R} & \rightarrow \mathbb{R} \\
s & \mapsto Q(s):=q s^{2}
\end{aligned}
$$

and for each real number $s$ in $[0,+\infty)$ define the following intervals of $\mathbb{R}$ :

$$
I_{s}:=\left[2^{Q(s)}, 2^{Q(s)}+Q(s+1)-Q(s)+\Xi\right)
$$

and

$$
J_{s}:=\left[2^{Q(s)}+Q(s+1)-Q(s)+\Xi, 2^{Q(s+1)}\right) .
$$

Denote by $\left(x_{j}\right)_{j=0}^{+\infty}$ the sequence in $\{0,1\}^{\mathbb{N}_{0}}$ defined by the property that $x_{j}=0$ if and only if there is an integer $s \geq 0$ such that $j+1$ is in $I_{s}$. Note that the first $\left|I_{0}\right|=q+\Xi$ entries of $\left(x_{j}\right)_{j=0}^{+\infty}$ are equal to 0 . Moreover, define the function $N: \mathbb{N}_{0} \rightarrow \mathbb{N}_{0}$, by $N(0):=0$, and for $k$ in $\mathbb{N}$ by

$$
N(k):=\sharp\left\{j \in\{0, \ldots, k-1\} \mid x_{j}=0\right\},
$$


and the function $B: \mathbb{N}_{0} \rightarrow \mathbb{N}_{0}$ by $B(0):=0, B(1):=1$, and for $k \geq 2$ by

$$
B(k):=1+\sharp\left\{j \in\{0, \ldots, k-2\} \mid x_{j} \neq x_{j+1}\right\} .
$$

Note that for $k \geq 1$ the number $B(k)$ is equal to the number of blocks of 0 's and 1's in the sequence $\left(x_{j}\right)_{j=0}^{k-1}$.

Observe that for every $s$ in $\mathbb{N}_{0}$ and every $k$ in $J_{s}$, we have

$$
N(k)=\sum_{j=0}^{s}\left|I_{j}\right|=\sum_{j=0}^{s}(Q(j+1)-Q(j)+\Xi)=Q(s+1)+\Xi \cdot(s+1)
$$

and

$$
B(k)=2(s+1) .
$$

On the other hand, for each $s$ in $\mathbb{N}_{0}$ and $k$ in $I_{s}$, we have

$$
N(k)=k-\left(2^{Q(s)}-1\right)+Q(s)+\Xi s
$$

and

$$
B(k)=2 s+1 .
$$

Lemma 4.1. The the following properties hold for each real number $s \geq 0$ :

(a) $2^{Q(s)}+Q(s+1)+\Xi \leq 2^{Q(s+1)-1}$.

(b) $\left|J_{s}\right| \geq 2^{Q(s+1)-1}$.

Proof. Part ( $a$ ) with $s=0$ is given by our hypothesis $2^{q-1} \geq q+1+\Xi$. For $s>0$, it follows from this and from the fact that the derivative of the function

$$
s \mapsto 2^{Q(s+1)-1}-\left(2^{Q(s)}+Q(s+1)+\Xi\right)
$$

is strictly positive on $[0,+\infty)$. Part $(b)$ follows easily from part $(a)$.

4.2. Estimates. Let $\Xi$ be a given integer and let $q, N$ and $B$ be as in the previous subsection. Given a real number $\xi$ such that $1 \leq \Xi-2 \xi \leq 2$, define the 2 variables series $\Pi$ on $[0,+\infty) \times[0,+\infty)$, by

$$
\Pi(\tau, \lambda):=\sum_{k=0}^{+\infty} 2^{-\lambda k-\tau N(k)+\tau \xi B(k)} .
$$

This subsection is dedicated to prove the following proposition.

Proposition B. For every $\tau \geq 1$ we have,

$$
\Pi(\tau, 0) \leq 2\left(2^{\tau \xi}+1\right) .
$$

Furthermore, for each $\tau$ in $\left(\frac{q-2}{q-1}, 1\right)$ we have

$$
\Pi\left(\tau, 2 \cdot 2^{-q\left(\frac{\Xi-2 \xi}{q(1-\tau)}-1\right)^{2}}\right) \leq 10 \cdot 2^{\tau \xi}+101,
$$

and for each $\Delta \geq 1$ we have

$$
2^{\Delta-4} \leq \Pi\left(\tau, 2^{-q\left(\frac{\Xi-2 \xi}{q(1-\tau)}+\Delta\right)^{2}}\right) .
$$

The proof of this proposition is at the end of this subsection.

For every real number $s$ in $[0,+\infty)$, define

$$
\lambda(s):=\frac{1}{\left|J_{s}\right|} \text {. }
$$

By part (b) of Lemma 4.1 and the hypothesis $q \geq 3$, we have $0<\lambda(s) \leq 1 / 4$. 
Lemma 4.2. The following properties hold:

1. For $\tau \geq 1$ we have

$$
\Pi(\tau, 0) \leq 2\left(2^{\tau \xi}+1\right) .
$$

2. For every real number $s$ in $[0,+\infty)$ and every $\tau$ in $(1 / 2,1)$ satisfying $\tau>$ $\frac{Q(s+1)-1}{Q(s+2)}$, we have

$$
\Pi(\tau, \lambda(s)) \leq 1+10 \cdot 2^{\tau \xi}+5 \sum_{j=0}^{\lfloor s\rfloor+1} 2^{(1-\tau) Q(j+1)-\tau(\Xi-2 \xi)(j+1)} .
$$

3. For every real number $s$ in $[0,+\infty)$ and every $\tau>0$, we have

$$
\frac{1}{8} 2^{(1-\tau) Q(\lfloor s\rfloor+1)-\tau(\Xi-2 \xi)(\lfloor s\rfloor+1)} \leq \Pi(\tau, \lambda(s)) .
$$

Proof. For $\tau>0, \lambda \geq 0$, and $s$ in $\mathbb{N}_{0}$, define

$$
I_{s}(\tau, \lambda):=\sum_{k \in I_{s}} 2^{-\lambda k-\tau N(k)+\tau \xi B(k)}
$$

and

$$
J_{s}(\tau, \lambda):=\sum_{k \in J_{s}} 2^{-\lambda k-\tau N(k)+\tau \xi B(k)},
$$

so that $\Pi(\tau, \lambda)=1+\sum_{s=0}^{+\infty} I_{s}(\tau, \lambda)+\sum_{s=0}^{+\infty} J_{s}(\tau, \lambda)$.

1. To prove part 1 , note that by (4.3), (4.4), and the hypothesis $\Xi-2 \xi \geq 1$, for every $\tau>0$ and every $\lambda \geq 0$ we have

$$
\begin{aligned}
\sum_{s=0}^{+\infty} I_{s}(\tau, \lambda) & \leq \sum_{s=0}^{+\infty} \sum_{m=1}^{\left|I_{s}\right|} 2^{-\tau(Q(s)+\Xi s+m)+\tau \xi \cdot(2 s+1)} \\
& =2^{\tau \xi} \sum_{s=0}^{+\infty} 2^{-\tau(Q(s)+(\Xi-2 \xi) s)} \sum_{m=1}^{\left|I_{s}\right|} 2^{-\tau m} \\
& \leq 2^{\tau \xi} \frac{2^{-\tau}}{1-2^{-\tau}} \sum_{s=0}^{+\infty} 2^{-\tau(\Xi-2 \xi) s} \\
& \leq 2^{\tau \xi} \frac{2^{-\tau}}{\left(1-2^{-\tau}\right)^{2}} .
\end{aligned}
$$

On the other hand, using (4.1), (4.2), the hypothesis $\Xi-2 \xi \geq 1$, and that for every $s \geq 0$ we have $\left|J_{s}\right| \leq 2^{Q(s+1)}$, we obtain for every $\tau \geq 1$

$$
\begin{aligned}
\sum_{s=0}^{+\infty} J_{s}(\tau, 0) & =\sum_{s=0}^{+\infty}\left|J_{s}\right| 2^{-\tau(Q(s+1)+\Xi(s+1))+2 \tau \xi \cdot(s+1)} \\
& \leq \sum_{s=0}^{+\infty} 2^{-(\tau-1) Q(s+1)-\tau(\Xi-2 \xi)(s+1)} \\
& \leq \frac{2^{-\tau}}{1-2^{-\tau}} .
\end{aligned}
$$

Combining inequalities (4.5) and (4.6), we get for every $\tau \geq 1$

$$
\Pi(\tau, 0) \leq 1+2^{\tau \xi} \frac{2^{-\tau}}{\left(1-2^{-\tau}\right)^{2}}+\frac{2^{-\tau}}{1-2^{-\tau}} \leq 2\left(2^{\tau \xi}+1\right) .
$$


This is part 1 of the lemma.

2. Fix $s$ in $[0,+\infty)$ and set $s_{0}:=\lfloor s\rfloor$. We use (4.5) to estimate $\Pi(\tau, \lambda(s))$. To estimate $\sum_{j=0}^{+\infty} J_{j}(\tau, \lambda(s))$, note that by definition of $\lambda(s)$, for each integer $\ell$ satisfying $1 \leq \ell \leq\left|J_{s}\right|$ we have

$$
\frac{1}{2} \leq 2^{-\lambda(s) \ell} \leq 1
$$

On the other hand, the hypothesis $q \geq 3$ implies that the function $j \mapsto\left|J_{j}\right|$ is nondecreasing on $[0,+\infty)$. Therefore, for each $j$ in $\left\{0, \ldots, s_{0}\right\}$ we have $\left|J_{j}\right| \leq\left|J_{s}\right|$ and then

$$
\frac{1}{2}\left|J_{j}\right| \leq \sum_{m=1}^{\left|J_{j}\right|} 2^{-\lambda(s) m} \leq\left|J_{j}\right|
$$

On the other hand

$$
\sum_{m=1}^{+\infty} 2^{-\lambda(s) m}=\frac{1}{2^{\lambda(s)}-1} \leq \frac{1}{\lambda(s) \log 2} \leq 2\left|J_{s}\right| .
$$

Note also that, by (4.1), (4.2), and the hypothesis $q+\Xi \geq 1$, for every $j$ in $\mathbb{N}_{0}$ we have by (4.8) and $\left|J_{s}\right| \leq 2^{Q(s+1)}$,

$$
\begin{aligned}
J_{j}(\tau, \lambda(s)) & =2^{-\tau(Q(j+1)+\Xi \cdot(j+1))+2 \tau \xi \cdot(j+1)} \sum_{k \in J_{j}} 2^{-\lambda(s) k} \\
& \leq 2\left|J_{s}\right| 2^{-\tau(Q(j+1)+(\Xi-2 \xi)(j+1))} \\
& \leq 2 \cdot 2^{Q(s+1)-\tau(Q(j+1)+(\Xi-2 \xi)(j+1))} .
\end{aligned}
$$

Taking $j=s_{0}+1$ and using the inequality $Q(s+1) \leq Q\left(s_{0}+2\right)$, we obtain,

$$
J_{s_{0}+1}(\tau, \lambda(s)) \leq 2 \cdot 2^{(1-\tau) Q\left(s_{0}+2\right)-\tau(\Xi-2 \xi)\left(s_{0}+2\right)} .
$$

On the other hand, our hypothesis $\tau \geq \frac{Q(s+1)-1}{Q(s+2)}$ implies that for $j \geq s_{0}+2$ we have

$$
Q(s+1)-\tau Q(j+1) \leq Q(s+1)-\tau Q(s+2) \leq 1 .
$$

So, using the hypothesis $\Xi-2 \xi \geq 1$ and summing (4.9) over $j$ satisfying $j \geq s_{0}+2$, we obtain

$$
\sum_{j=s_{0}+2}^{+\infty} J_{j}(\tau, \lambda(s)) \leq \sum_{j=s_{0}+2}^{+\infty} 2^{2-\tau(\Xi-2 \xi)(j+1)} \leq \frac{2^{2-3(\Xi-2 \xi) \tau}}{1-2^{-\tau}} .
$$

Now we complete the estimate of $\sum_{j=0}^{+\infty} J_{j}(\tau, \lambda(s))$, by estimating the terms for which $j$ is in $\left\{0, \ldots, s_{0}\right\}$. From (4.7), the first equality in (4.9), and $\left|J_{j}\right| \leq 2^{Q(j+1)}$, we deduce that for every integer $j$ in $\left\{0, \ldots, s_{0}\right\}$ we have

$$
J_{j}(\tau, \lambda(s)) \leq\left|J_{j}\right| \cdot 2^{-\tau(Q(j+1)+(\Xi-2 \xi)(j+1))} \leq 2^{(1-\tau) Q(j+1)-\tau(\Xi-2 \xi)(j+1)} .
$$


Summing over $j$ in $\left\{0, \ldots, s_{0}\right\}$ and using inequalities (4.10) and (4.11), we obtain

$$
\begin{aligned}
& \sum_{j=0}^{+\infty} J_{j}(\tau, \lambda(s)) \\
& \leq \sum_{j=0}^{s_{0}} 2^{(1-\tau) Q(j+1)-\tau(\Xi-2 \xi)(j+1)}+2 \cdot 2^{(1-\tau) Q\left(s_{0}+2\right)-\tau(\Xi-2 \xi)\left(s_{0}+2\right)}+\frac{2^{2-3(\Xi-2 \xi) \tau}}{1-2^{-\tau}} \\
& \leq 2 \sum_{j=0}^{s_{0}+1} 2^{(1-\tau) Q(j+1)-\tau(\Xi-2 \xi)(j+1)}+\frac{2^{2-3(\Xi-2 \xi) \tau}}{1-2^{-\tau}} .
\end{aligned}
$$

Together with (4.5) this implies

$$
\begin{aligned}
& \Pi(\tau, \lambda(s)) \leq 1+\frac{2^{-\tau}}{\left(1-2^{-\tau}\right)^{2}} 2^{\tau \xi} \\
& \quad+2 \sum_{j=0}^{s_{0}+1} 2^{(1-\tau) Q(j+1)-\tau(\Xi-2 \xi)(j+1)}+\frac{2^{2-3(\Xi-2 \xi) \tau}}{1-2^{-\tau}} .
\end{aligned}
$$

Using the hypothesis that $\tau$ is in $(1 / 2,1)$, we have $\frac{2^{-\tau}}{\left(1-2^{-\tau}\right)^{2}} \leq 10$. Using in addition the hypotheses $q \geq 3$ and $\Xi-2 \xi \geq 1$, we have

$$
\begin{aligned}
& \frac{2^{2-3(\Xi-2 \xi) \tau}}{1-2^{-\tau}} \leq 3 \cdot 2^{3-(\Xi-2 \xi+3) \tau} \leq 3 \cdot 2^{(1-\tau) Q(1)}-(\Xi-2 \xi) \tau \\
& \leq 3 \sum_{j=0}^{s_{0}+1} 2^{(1-\tau) Q(j+1)-\tau(\Xi-2 \xi)(j+1)}
\end{aligned}
$$

We obtain part 2 of the lemma by combining these estimates with (4.12).

3. Fix $s$ in $[0,+\infty)$ and set $s_{0}:=\lfloor s\rfloor$. By part (b) of Lemma 4.1$]$ and the definition of $\lambda(s)$, for each $s$ in $[0,+\infty)$ we have

$$
\lambda(s)=\left|J_{s}\right|^{-1} \leq \frac{1}{2^{Q(s+1)-1}} .
$$

From this inequality and from part (a) of Lemma 4.1 we obtain that for every integer $j$ in $\left\{0, \ldots, s_{0}\right\}$ we have

$$
\begin{aligned}
& \lambda(s)\left(2^{Q(j)}+Q(j+1)-Q(j)+\Xi-1\right) \\
& \leq \lambda(s)\left(2^{Q(j)}+Q(j+1)+\Xi\right) \\
& \leq \frac{2^{Q(s)}+Q(s+1)+\Xi}{2^{Q(s+1)-1}} \\
& \leq 1 .
\end{aligned}
$$

In view of part (b) of Lemma 4.1, formulas (4.1) and (4.2), the first inequality of (4.7), the first equality in (4.9), and the hypothesis $q+\Xi \geq 1$, we deduce that 
for $j=s_{0}$ we have

(4.14)

$$
\begin{aligned}
& \frac{1}{8} 2^{(1-\tau) Q\left(s_{0}+1\right)-\tau(\Xi-2 \xi)\left(s_{0}+1\right)} \\
& \leq \frac{1}{4}\left|J_{s_{0}}\right| 2^{-\tau\left(Q\left(s_{0}+1\right)+\Xi \cdot\left(s_{0}+1\right)\right)+2 \tau \xi \cdot\left(s_{0}+1\right)} \\
& \leq \frac{1}{2}\left|J_{s_{0}}\right| 2^{-\lambda(s)\left(2^{Q\left(s_{0}\right)}+Q\left(s_{0}+1\right)-Q\left(s_{0}\right)+\Xi-1\right)-\tau\left(Q\left(s_{0}+1\right)+\Xi \cdot\left(s_{0}+1\right)\right)+2 \tau \xi \cdot\left(s_{0}+1\right)} \\
& \leq\left(\sum_{m=1}^{\left|J_{s_{0}}\right|} 2^{-\lambda(s) m}\right) 2^{-\lambda(s)\left(2^{Q\left(s_{0}\right)}+Q\left(s_{0}+1\right)-Q\left(s_{0}\right)+\Xi-1\right)-\tau\left(Q\left(s_{0}+1\right)+\Xi \cdot\left(s_{0}+1\right)\right)+2 \tau \xi \cdot\left(s_{0}+1\right)} \\
& =J_{s_{0}}(\tau, \lambda(s)) .
\end{aligned}
$$

This proves part 3 of the lemma and completes the proof.

Define the function $s:(-\infty, 1) \rightarrow \mathbb{R}$ by

$$
s(\tau)=\frac{\Xi-2 \xi}{q(1-\tau)} .
$$

Lemma 4.3. For every $\tau$ in $\left(\frac{q-2}{q-1}, 1\right)$, we have

$$
\Pi(\tau, \lambda(s(\tau)-2)) \leq 10 \cdot 2^{\tau \xi}+101,
$$

and for every $\Omega \geq 0$, we have

$$
2^{\Omega-3} \leq \Pi(\tau, \lambda(s(\tau)+\Omega))
$$

Proof. Fix $\tau$ in $\left(\frac{q-2}{q-1}, 1\right)$ and $\Omega \geq 0$. Note that the inequality $\tau>\frac{q-2}{q-1}$ implies $\tau>$ $\frac{q-4}{q}$. Moreover, this last inequality is equivalent to

$$
\tau>\frac{Q\left(\frac{2}{q(1-\tau)}-1\right)-1}{Q\left(\frac{2}{q(1-\tau)}\right)} .
$$

On the other hand, the function $s \mapsto \frac{Q(s-1)-1}{Q(s)}$ is strictly increasing on $\left(\frac{q-1}{q},+\infty\right)$. Since the inequalities $1 \leq \Xi-2 \xi$ and $\tau>\frac{q-2}{q-1}$ imply $s(\tau)>\frac{q-1}{q}$, using $\Xi-2 \xi \leq 2$ we deduce

$$
\frac{Q(s(\tau)-1)-1}{Q(s(\tau))} \leq \frac{Q\left(\frac{2}{q(1-\tau)}-1\right)-1}{Q\left(\frac{2}{q(1-\tau)}\right)}<\tau
$$

So the hypotheses of part 2 of Lemma 4.2 are satisfied with $s=s(\tau)-2$. Let $F$ : $\mathbb{R} \rightarrow \mathbb{R}$ be the quadratic function defined by

$$
F(\ell):=(1-\tau) Q(\ell)-\tau(\Xi-2 \xi) \ell .
$$

Note that $F(0)=0$,

$$
F\left(\frac{s(\tau)}{2}\right)=\frac{(\Xi-2 \xi)^{2}}{2 q}-\frac{\Xi-2 \xi}{2}\left(\frac{s(\tau)}{2}\right) \text { and } F(s(\tau))=\frac{(\Xi-2 \xi)^{2}}{q}
$$


Using that $F$ is convex, we conclude that for each $\ell$ in $[0, s(\tau)]$ we have

$$
\begin{aligned}
& F(\ell)=(1-\tau) Q(\ell)-\tau(\Xi-2 \xi) \ell \\
& \quad \leq \frac{(\Xi-2 \xi)^{2}}{q}-\frac{\Xi-2 \xi}{2} \min \{\ell, s(\tau)-\ell\} .
\end{aligned}
$$

Therefore, putting $s^{+}=s(\tau)-2$ and using $1 \leq \Xi-2 \xi \leq 2$ and $q \geq 3$, we have

$$
\begin{aligned}
\sum_{j=0}^{\left\lfloor s^{+}\right\rfloor+1} 2^{(1-\tau) Q(j+1)-\tau(\Xi-2 \xi)(j+1)} \leq 2 \sum_{\ell=0}^{\left\lfloor s^{+}\right\rfloor+2} 2^{\frac{(\Xi-2 \xi)^{2}}{q}-\frac{\Xi-2 \xi}{2} \ell} & \\
& \leq 2 \cdot 2^{\frac{4}{q}} \frac{1}{1-2^{-\frac{1}{2}}} \leq 20 .
\end{aligned}
$$

The first inequality of the lemma is then obtained using part 2 of Lemma 4.2 with $s=s^{+}$.

To prove the second inequality, note that

$$
F(s(\tau)+\Omega)=\frac{(\Xi-2 \xi)^{2}}{q}+(\Xi-2 \xi)(2-\tau) \Omega+q(1-\tau) \Omega^{2} \geq(\Xi-2 \xi) \Omega \geq \Omega
$$

and that $F$ is increasing on the interval $\left[\frac{\tau}{2} \frac{\Xi-2 \xi}{q(1-\tau)},+\infty\right)$, that contains $s(\tau)$. So, if we put $s^{-}=s(\tau)+\Omega$, then

$$
\Omega \leq F\left(s^{-}\right) \leq F\left(\left\lfloor s^{-}\right\rfloor+1\right)=(1-\tau) Q\left(\left\lfloor s^{-}\right\rfloor+1\right)-\tau(\Xi-2 \xi)\left(\left\lfloor s^{-}\right\rfloor+1\right) .
$$

Together with part 3 of Lemma 4.2 with $s=s^{-}$, we obtain

$$
2^{\Omega} \leq 2^{(1-\tau) Q\left(\left\lfloor s^{-}\right\rfloor+1\right)-\tau(\Xi-2 \xi)\left(\left\lfloor s^{-}\right\rfloor+1\right)} \leq 8 \Pi\left(\tau, \lambda\left(s^{-}\right)\right),
$$

from which we obtain the second inequality of the lemma.

Proof of Proposition B. The first inequality is part 1 of Lemma 4.2. To prove the other inequalities, note that by the definition of $\lambda(s)$ we have $\lambda(s) \geq 2^{-Q(s+1)}$. On the other hand, by part (b) of Lemma 4.1 we have $\lambda(s) \leq 2 \cdot 2^{-Q(s+1)}$. So, using the definition of the function $s$ we have for each $\tau$ in $(0,1)$ and $\Delta \geq 1$,

$$
\lambda(s(\tau)-2) \leq 2 \cdot 2^{-q\left(\frac{\Xi-2 \xi}{q(1-\tau)}-1\right)^{2}}
$$

and

$$
\lambda(s(\tau)+\Delta-1) \geq 2^{-q\left(\frac{\Xi-2 \xi}{q(1-\tau)}+\Delta\right)^{2}} .
$$

Then the desired inequalities are a direct consequence of Lemma 4.3 with $\Omega=\Delta-1$ and of the fact that for a fixed $\tau$ the function

$$
\lambda \mapsto \Pi(\tau, \lambda)
$$

is nonincreasing on the set where it is finite.

\section{Estimating the GeOMETRIC PRESSURE FUnCTION}

In this section we prove the Main Technical Theorem. In $\$ 5.1$ we show a general result about conformal measures, and in $\$ 5.2$ we make some technical estimates (Proposition 5.2). The proof of Main Technical Theorem is in 55.3, after recalling a few results from CRL12. 
5.1. Conformal measures. Recall that, given an integer $n \geq 3$ and a parameter $c$ in $\mathcal{K}_{n}$, the conical or radial Julia set of $\left.f_{c}\right|_{I_{c}}$ (resp. $f_{c}$ ) is the set of all points $x$ in $I_{c}$ (resp. $J_{c}$ ) for which the following property holds: There exists $r>0$ and an unbounded sequence of positive integers $\left(n_{j}\right)_{j=1}^{+\infty}$, such that for every $j$ the map $\left.f_{c}\right|_{I_{c}} ^{n_{j}}\left(\operatorname{resp} . f_{c}^{n_{j}}\right)$ maps a neighborhood of $x$ in $I_{c}$ (resp. $J_{c}$ ) diffeomorphically to $B\left(f_{c}^{n_{j}}(x), r\right)$.

Proposition 5.1. Let $n \geq 4$ be an integer, $c$ a parameter in $\mathcal{K}_{n}$, and let $t>0$ and $p$ in $\mathbb{R}$ be given. Then there is at most one $(t, p)$-conformal probability measure of $\left.f_{c}\right|_{I_{c}}$ (resp. $f_{c}$ ) supported on $I_{c}$ (resp. $\left.J_{c}\right)$. If such a measure $\mu$ exists, then $p \geq P_{c}^{\mathbb{R}}(t)$ (resp. $\left.p \geq P_{c}^{\mathbb{C}}(t)\right)$, and $\mu$ is either supported on the backward orbit of 0 and dissipative, or $\mu$ is nonatomic and supported on the conical Julia set of $\left.f_{c}\right|_{I_{c}}$ (resp. $f_{c}$ ). Furthermore, the former case holds precisely when following series converges:

$$
\begin{aligned}
\sum_{j=1}^{+\infty} \exp (-j p) & \sum_{\left.y \in f_{c}\right|_{I_{c}} ^{-j}(0)}\left|D f_{c}^{j}(y)\right|^{-t} \\
& \left(\operatorname{resp} . \sum_{j=1}^{+\infty} \exp (-j p) \sum_{y \in f_{c}^{-j}(0)}\left|D f_{c}^{j}(y)\right|^{-t}\right) .
\end{aligned}
$$

Proof. By [Urb03, Theorem 4.2] the conical Julia set of $f_{c}$ is the complement in $J_{c}$ of the backward orbit of $z=0$. This implies that the conical Julia set of $\left.f_{c}\right|_{I_{c}}$ contains the complement in $I_{c}$ of the backward orbit of $z=0$ under $\left.f_{c}\right|_{I_{c}}$. On the other hand, this last set is clearly disjoint from the conical Julia set of $\left.f_{c}\right|_{I_{c}}$, so this proves that the conical Julia set of $\left.f_{c}\right|_{I_{c}}$ is the complement in $I_{c}$ of the backward orbit of $z=0$.

Let $\mu$ be a $(t, p)$-conformal probability measure for $\left.f_{c}\right|_{I_{c}}$ (resp. $f_{c}$ ) supported on $I_{c}\left(\operatorname{resp} . J_{c}\right)$. If $\mu$ is supported on the backward orbit of $z=0$, then it is uniquely determined by the mass it assigns to $z=0$, and therefore it is unique up to a scalar factor. Note moreover that in this case $\mu$ is dissipative, because it charges the wandering set $\{0\}$. If $\mu$ is not entirely supported on the backward orbit of $z=0$, then it charges the conical Julia set, so $\mu$ is nonatomic, it is supported on the conical Julia set and it is the unique $(t, p)$-conformal measure of $\left.f_{c}\right|_{I_{c}}$ (resp. $f_{c}$ ) supported on $I_{c}$ (resp. $J_{c}$ ), up to a scalar factor, see [PRL11, Proposition 4.1] for the complex case; the proof of the uniqueness part of this result applies without change to the real case. This completes the proof that $\mu$ is unique.

To prove that in the complex case we have $p \geq P_{c}^{\mathbb{C}}(t)$, let $\delta>0$ be sufficiently small so that $B(0,2 \delta)$ is disjoint from the forward orbit of the critical point. It follows that there is a constant $K>1$ such that for every integer $j \geq 1$ and every $y$ in $f_{c}^{-j}(0)$, the map $f_{c}^{j}$ maps a neighborhood $W_{y}$ of $y$ biholomorphically to $B(0, \delta)$ with distortion bounded by $K$. Therefore,

$$
\mu\left(W_{y}\right) \geq K^{-t} \exp (-j p)\left|D f_{c}^{j}(y)\right|^{-t} \mu(B(0, \delta)) .
$$

So, if we put $C:=K^{-1} \mu(B(0, \delta))>0$, then for every integer $j \geq 1$ we have

$$
1 \geq \sum_{y \in f_{c}^{-j}(0)} \mu\left(W_{y}\right) \geq C \exp (-j p) \sum_{y \in f_{c}^{-j}(0)}\left|D f_{c}^{j}(y)\right|^{-t}
$$


Since by [PRLS04, Theorem A] we have

$$
\lim _{j \rightarrow+\infty} \frac{1}{j} \log \sum_{y \in f_{c}^{-j}(0)}\left|D f_{c}^{j}(y)\right|^{-t}=P_{c}^{\mathbb{C}}(t),
$$

this proves $p \geq P_{c}^{\mathbb{C}}(t)$.

To prove that in the real case we have $p \geq P_{c}^{\mathbb{R}}(t)$, we note that the proof of [PRLS04, Proposition 2.1] can be adapted to show

$$
\lim _{j \rightarrow+\infty} \frac{1}{j} \log \sum_{y \in\left(f_{c}||_{I_{c}}\right)^{-j}(0)}\left|D f_{c}^{j}(y)\right|^{-t}=P_{c}^{\mathbb{R}}(t),
$$

using the fact that $z=0$ is not in the closure of the orbit of the critical value of $f_{c}$. The rest of the proof of $p \geq P_{c}^{\mathbb{R}}(t)$ is similar to the proof above.

To prove the last statement, observe first that if there is a $(t, p)$-conformal measure for $\left.f_{c}\right|_{I_{c}}$ (resp. $f_{c}$ ) that is supported on the backward orbit of $z=0$, then its total mass is equal to (5.1) times the mass at $z=0$. This proves that (5.1) is finite. Conversely, if (5.1) is finite, then

$$
\begin{aligned}
\delta_{0}+\sum_{j=1}^{+\infty} \sum_{\left.y \in f_{c}\right|_{I_{c}} ^{-j}(0)} \exp (-j p)\left|D f_{c}^{j}(y)\right|^{-t} \delta_{y} & \left(\text { resp. } \delta_{0}+\sum_{j=1}^{+\infty} \sum_{y \in f_{c}^{-j}(0)} \exp (-j p)\left|D f_{c}^{j}(y)\right|^{-t} \delta_{y}\right) .
\end{aligned}
$$

is finite and it is a $(t, p)$-conformal measure for $\left.f_{c}\right|_{I_{c}}$ (resp. $f_{c}$ ) supported on $I_{c}$ $\left(\right.$ resp. $J_{c}$ ).

5.2. Phase transition parameter. Recall that for each parameter $c$ in $\mathcal{P}_{5}(-2)$, we have put

$$
\theta(c)=\left|\frac{D g_{c}(p(c))}{D g_{c}(\widetilde{p}(c))}\right|^{1 / 2} \text { and } \xi(c)=-\frac{\log (\zeta(c) \widetilde{\zeta}(c))}{4 \log \theta(c)} .
$$

Put $t(c):=\frac{\log 2}{\log \theta(c)}$ and for every integer $n \geq 5$ put

$$
\xi_{n}:=\sup _{c \in \mathcal{K}_{n}} \xi(c) .
$$

This subsection is dedicated to prove the following estimates, used in the proof of the Main Technical Theorem.

Proposition 5.2. There is an integer $n_{2} \geq 5$ such that for every integer $n \geq$ $n_{2}$, and every $c$ in $\mathcal{K}_{n}$, we have $\left\lceil 2 \xi_{n}+1\right\rceil-2 \xi(c) \leq 2$. Furthermore, for every constant $T>0$ there is $n_{3} \geq 5$ such that for every integer $n \geq n_{3}$ and every parameter $c$ in $\mathcal{K}_{n}$, we have $t(c) \geq T$.

The proof of this proposition is at the end of this subsection and it follows from the following sequence of lemmas.

Lemma 5.3 (CRL12, Lemma A.1). We have,

$$
\left.\frac{\partial}{\partial c}\left|D f_{c}^{3}(p(c))\right|\right|_{c=-2}>\left.\frac{\partial}{\partial c}\left|D f_{c}^{3}(\widetilde{p}(c))\right|\right|_{c=-2} .
$$


Lemma 5.4. We have $\theta(-2)=1$ and $D \theta(-2)>0$.

Proof. For $c=-2$,

$$
\{2 \cos (2 \pi / 7), 2 \cos (4 \pi / 7), 2 \cos (6 \pi / 7)\}
$$

and

$$
\{2 \cos (2 \pi / 9), 2 \cos (4 \pi / 9), 2 \cos (8 \pi / 9)\}
$$

are the only orbits of minimal period 3 of $f_{-2}$. Thus, we have

$$
\left|D f_{-2}^{3}(p(-2))\right|=\left|D f_{-2}^{3}(\widetilde{p}(-2))\right|=8 \text {, and } \theta(-2)=1 .
$$

Together with Lemma 5.3 , we obtain $D \theta(-2)>0$.

Lemma 5.5. We have $\zeta(-2) \cdot \widetilde{\zeta}(-2)=1$, and the function $\xi(c)$ is real analytic at $c=-2$.

Proof. Let $c$ be a parameter in $\mathcal{P}_{5}(-2)$. For every integer $m \geq 1$ denote by $p_{m}(c)$ the periodic point in $\Lambda_{c}$ whose itinerary consists of the periodic sequence whose period is the concatenation of $m$ consecutive 0's and of $m$ consecutive 1's. By Proposition 3.1

$$
\frac{D g_{c}^{2 m}\left(p_{m}(c)\right)}{\left(D g_{c}(p(c)) D g_{c}(\widetilde{p}(c))\right)^{m}} \rightarrow \zeta(c) \widetilde{\zeta}(c) \quad \text { as } m \rightarrow+\infty .
$$

On the other hand, using the identity $f_{-2}(2 \cos (x))=2 \cos (2 x)$ for $x$ in $\mathbb{R}$, we obtain

$$
\frac{D g_{-2}^{2 m}\left(p_{m}(-2)\right)}{\left(D g_{-2}(p(-2)) D g_{-2}(\widetilde{p}(-2))\right)^{m}}=1 \text {. }
$$

This proves $\zeta(-2) \cdot \widetilde{\zeta}(-2)=1$. To prove that $\xi$ is real analytic at $c=-2$, notice that each of the functions $\theta, \zeta$, and $\widetilde{\zeta}$ is real analytic at $c=-2$ (Proposition 3.1). Since $\zeta(-2) \cdot \widetilde{\zeta}(-2)=1$ and $\theta(-2)=1$ (Lemma 5.4), we have that the functions $A$ and $B$ defined for $c$ in $\mathcal{P}_{5}(-2)$ by

$$
A(c):=\log (\zeta(c) \widetilde{\zeta}(c)) /(c+2) \text { and } B(c):=\log \theta(c) /(c+2),
$$

are also real analytic at $c=-2$. Moreover, $B(-2) \neq 0$ since by Lemma 5.4 we have $D \theta(-2) \neq 0$. Thus, the quotient $4 \xi(c)=A(c) / B(c)$ is real analytic at $c=-2$. This concludes the proof of the lemma.

Proof of Proposition 5.2. By Lemma 5.4, there is $\delta>0$ such that for every $c$ in $(-2,-2+\delta)$ we have

$$
1<\theta(c)<2^{1 / T} .
$$

On the other hand, by Proposition [2.6, there is an integer $n_{0} \geq 3$ such that for every $n \geq n_{0}$ we have $\mathcal{K}_{n} \subset(-2,-2+\delta)$. These assertions imply the second part of the proposition.

To prove the first part, notice that by Lemma 5.5 there is $\epsilon>0$ such that $\xi(c)$ is uniformly continuous on the interval $[-2,-2+\epsilon]$. By Proposition 2.6 for every sufficiently large integer $n$ we have $\mathcal{K}_{n} \subset[-2,-2+\epsilon]$ and moreover the diameter of $\mathcal{K}_{n}$ converges to 0 as $n \rightarrow+\infty$. Thus, for every sufficiently large $n$ we have

$$
\xi_{n}-\xi(c)<\frac{1}{2} .
$$

This implies the first assertion of the proposition and concludes the proof. 
5.3. Proof of the Main Technical Theorem. We start recalling some results from [CRL12.

Proposition 5.6 (CRL12, Proposition D). There is an integer $n_{5} \geq 4$ and a constant $C_{3}>1$, such that for every integer $n \geq n_{5}$ and every parameter $c$ in $\mathcal{K}_{n}$, the following properties hold for each $t \geq 3$ :

1. For $p$ in $\left[-t \chi_{\text {crit }}(c) / 2,0\right)$ satisfying

$$
\sum_{k=0}^{+\infty} \exp (-(n+3 k) p)\left|D f_{c}^{n+3 k}(c)\right|^{-t / 2} \geq C_{3}^{t},
$$

we have $\mathscr{P}_{c}^{\mathbb{R}}(t, p)>0$ and $P_{c}^{\mathbb{R}}(t) \geq p$.

2. For $p \geq-t \chi_{\text {crit }}(c) / 2$ satisfying

$$
\sum_{k=0}^{+\infty} \exp (-(n+3 k) p)\left|D f_{c}^{n+3 k}(c)\right|^{-t / 2} \leq C_{3}^{-t}
$$

we have $\mathscr{P}_{c}^{\mathbb{C}}(t, p)<0$ and $P_{c}^{\mathbb{C}}(t) \leq p$.

Lemma 5.7 (CRL12, Proposition 6.2). For every integer $n \geq 5$, every parameter $c$ in $\mathcal{K}_{n}$, and every $t>0$, we have

$$
P_{c}^{\mathbb{C}}(t) \geq P_{c}^{\mathbb{R}}(t) \geq-t \chi_{\text {crit }}(c) / 2 .
$$

Lemma 5.8 (CRL12, Lemma 6.5). There is $n_{6} \geq 5$, such that for every integer $n \geq n_{6}$, every $c$ in $\mathcal{K}_{n}$, and every $t \geq 3$ and

$$
p \geq P_{c}^{\mathbb{R}}(t)\left(\text { resp. } p \geq P_{c}^{\mathbb{C}}(t)\right)
$$

satisfying $\mathscr{P}_{c}^{\mathbb{R}}(t, p)<0$ (resp. $\mathscr{P}_{c}^{\mathbb{C}}(t, p)<0$ ), the sum (5.1) is finite.

Proof of the Main Technical Theorem. Let $C_{2}$ and $v_{2}$ be the constants given by Proposition $\mathrm{A} n_{5}$ and $C_{3}$ the constants given by Proposition 5.6. and $n_{6}$ the constant given by Lemma 5.8. Since for $c=-2$ we have

$$
\left|D g_{-2}(\widetilde{p}(-2))\right|^{1 / 3}=2 \text { and }\left|D f_{-2}(\beta(-2))\right|=4 \text {, }
$$

there is $\delta>0$ such that that for each $c$ in $(-2,-2+\delta)$ we have

$$
\frac{\left|D g_{c}(\widetilde{p}(c))\right|^{1 / 3}}{\left|f_{c}(\beta(c))\right|}<\frac{2}{3} \text {. }
$$

By Proposition 2.6 there is $n_{0} \geq 3$ such that for every integer $n \geq n_{0}$ the set $\mathcal{K}_{n}$ is contained in $(-2,-2+\delta)$; thus for every $c$ in $\mathcal{K}_{n}$ we have (5.2). Since the closure of $\mathcal{P}_{6}(-2)$ is contained in $\mathcal{P}_{5}(-2)$ (part 1 of Lemma 2.3), by Proposition 3.1 we have,

$$
Z:=\sup _{c \in \mathcal{P}_{6}(-2)}-\frac{\log (\zeta(c) \widetilde{\zeta}(c))}{4 \log 2}<+\infty .
$$

Fix $n \geq \max \left\{6, n_{0}, n_{5}, n_{6}\right\}$ large enough such that

$$
C_{2}\left(\frac{2}{3}\right)^{\frac{1}{2} n}\left(10 \cdot 2^{Z}+101\right)<C_{3}^{-1} .
$$

In view of Proposition 5.2. we can take $n$ larger if necessary so that for every $c$ in $\mathcal{K}_{n}$ we have

$$
t(c)=\frac{\log 2}{\log \theta(c)} \geq 6
$$


and such that, if we put

$$
\Xi:=\left\lceil 2 \xi_{n}+1\right\rceil,
$$

then for every $c$ in $\mathcal{K}_{n}$ we have $\Xi-2 \xi(c) \leq 2$. Consider the sequence $\left(x_{j}\right)_{j=0}^{+\infty}$ in $\{0,1\}^{\mathbb{N}_{0}}$ defined in 4.1 for this value of $\Xi$ and for some integer $q \geq 3$ satisfying in addition,

$$
q+\Xi \geq 1 \text { and } 2^{q-1} \geq q+1+\Xi .
$$

By Proposition 2.6. there is a parameter $c$ in $\mathcal{K}_{n}$ such that $\iota(c)=\left(x_{j}\right)_{j=0}^{+\infty}$. Finally, put $t_{*}:=t(c)$, and fix $\Delta \geq 1$ sufficiently large such that

$$
C_{2}^{-t_{*}} \exp (-n)\left(\frac{\exp \left(\chi_{\text {crit }}(c)\right)}{\left|D f_{c}(\beta(c))\right|}\right)^{\frac{t_{*}}{2} n} 2^{\Delta-4}>C_{3}^{t_{*}}
$$

Put

$$
t_{0}:=\frac{q-2}{q-1} t_{*}, \xi:=\xi(c), \text { and } \kappa:=\Xi-2 \xi,
$$

and define the functions

$$
\delta^{+}, \delta^{-}, p^{+}, p^{-}:\left(t_{0},+\infty\right) \rightarrow \mathbb{R}
$$

as in the statement of the Main Technical Theorem. Taking $\Delta$ larger if necessary, assume that for every $t$ in $\left(t_{0},+\infty\right)$ we have $p^{-}(t)<0$.

We start showing that $c$ satisfies the hypotheses of Proposition A, By (4.1) and (4.3), we have

$$
\frac{N_{c}(k)}{k} \rightarrow 0 \text { as } k \rightarrow+\infty \text {. }
$$

Denote by $\left(m_{j}\right)_{j=0}^{+\infty}$ the sequence of lengths of the blocks of 0's and 1's in $\iota(c)$. So, using the notation in 4.1 for every integer $s \geq 0$ we have $m_{2 s}=\left|I_{s}\right|$ and $m_{2 s+1}=$ $\left|J_{s}\right|$. By part (a) of Lemma 4.1 we have for every integer $s \geq 0$

$$
\min \left\{m_{2 s}, m_{2 s+1}\right\}=\min \left\{\left|I_{s}\right|,\left|J_{s}\right|\right\}=\left|I_{s}\right|=q(2 s+1)+\Xi,
$$

and for every integer $s \geq 1$

$$
\min \left\{m_{2 s+1}, m_{2 s+2}\right\}=\min \left\{\left|J_{s}\right|,\left|I_{s+1}\right|\right\}=\left|I_{s+1}\right|=q(2 s+3)+\Xi .
$$

Thus

$$
\sum_{j=2}^{+\infty} \exp \left(-\min \left\{m_{j}, m_{j+1}\right\} v_{2}\right) \leq 2 \sum_{s=1}^{+\infty} \exp \left(-(q(2 s+1)+\Xi) v_{2}\right)<+\infty .
$$

This proves $c$ satisfies the hypotheses of Proposition $\mathrm{A}$.

Note that by our choice of $n$ and the hypothesis $q \geq 3$, we have

$$
t_{0}=\frac{q-2}{q-1} t_{*} \geq \frac{1}{2} t_{*} \geq 3 .
$$

On the other hand, by (5.5) and Lemma 3.5 we have

$$
\exp \left(\chi_{\text {crit }}(c)\right)=\left|D g_{c}(\widetilde{p}(c))\right|^{1 / 3} \text {. }
$$

In particular, $\chi_{\text {crit }}(c)>0$. Consider the 2 variables series $\Pi$ defined as in $\$ 4.2$ for the above choices of $\Xi, q$, and $\xi$, and note that it coincides with the 2 variables series $\Pi_{c}$ defined in 33.3 for our choice of the parameter $c$.

To prove that for every $t>t_{0}$ we have $P_{c}^{\mathbb{R}}(t) \geq p^{-}(t)$, note first that when $t \geq t_{*}$ this is given by Lemma 5.7. On the other hand, from Propositions $\mathrm{A}$ and $\mathrm{B}$, (5.2), 
(5.4), (5.6), and from the fact that for every $t$ in $\left(t_{0}, t_{*}\right)$ we have $\delta_{-}(t) \leq 1$, we deduce

$$
\begin{aligned}
& \sum_{k=0}^{+\infty} \exp \left(-(n+3 k) p^{-}(t)\right)\left|D f_{c}^{n+3 k}(c)\right|^{-\frac{t}{2}} \\
& =\sum_{k=0}^{+\infty} \exp \left(-(n+3 k)\left(-t \frac{\chi_{\text {crit }}(c)}{2}+\delta^{-}(t)\right)\right)\left|D f_{c}^{n+3 k}(c)\right|^{-\frac{t}{2}} \\
& \geq C_{2}^{-t} \exp \left(-n \delta^{-}(t)\right)\left(\frac{\exp \left(\chi_{\text {crit }}(c)\right)}{\left|D f_{c}(\beta(c))\right|}\right)^{\frac{t}{2} n} \Pi\left(t \frac{\log \theta(c)}{\log 2}, \frac{3 \delta^{-}(t)}{\log 2}\right) \\
& \geq C_{2}^{-t} \exp \left(-n \delta^{-}(t)\right)\left(\frac{\exp \left(\chi_{\text {crit }}(c)\right)}{\left|D f_{c}(\beta(c))\right|}\right)^{\frac{t}{2} n} 2^{\Delta-4} \\
& >C_{3}^{t_{*}} \\
& \geq C_{3}^{t} .
\end{aligned}
$$

Since for each $t$ in $\left(t_{0}, t_{*}\right)$ we have $p^{-}(t)<0$, the inequality above combined with part 1 of Proposition [5.6 implies that for every $t$ in $\left(t_{0}, t_{*}\right)$ we have $P_{c}^{\mathbb{R}}(t) \geq p^{-}(t)$.

Now we turn to the proof that for every $t>t_{0}$ we have $P_{c}^{\mathbb{C}}(t) \leq p^{+}(t)$ and that for every $t \geq t_{*}$ we have $\mathscr{P}_{c}^{\mathbb{C}}\left(t,-t \frac{\chi_{\text {crit }}(c)}{2}\right)<0$. Combining Propositions $\mathrm{A}$ and $\mathrm{B}$ using the definition of $\xi=\xi(c)$ and $Z$, and using (5.2), (5.3) and (5.6), we deduce that for every $t \geq t_{*}$

$$
\begin{aligned}
& \sum_{k=0}^{+\infty} \exp \left(-(n+3 k) p^{+}(t)\right)\left|D f_{c}^{n+3 k}(c)\right|^{-\frac{t}{2}} \\
& =\sum_{k=0}^{+\infty} \exp \left(-(n+3 k)\left(-t \frac{\chi_{\text {crit }}(c)}{2}\right)\right)\left|D f_{c}^{n+3 k}(c)\right|^{-\frac{t}{2}} \\
& \leq C_{2}^{t}\left(\frac{\exp \left(\chi_{\text {crit }}(c)\right)}{\left|D f_{c}(\beta(c))\right|}\right)^{\frac{t}{2} n} \Pi\left(t \frac{\log \theta(c)}{\log 2}, 0\right) \\
& \leq C_{2}^{t}\left(\frac{\exp \left(\chi_{\text {crit }}(c)\right)}{\left|D f_{c}(\beta(c))\right|}\right)^{\frac{t}{2} n} 2\left(2^{t \frac{\log \theta(c)}{\log 2} \xi}+1\right) \\
& \leq C_{2}^{t}\left(\frac{\exp \left(\chi_{\text {crit }}(c)\right)}{\left|D f_{c}(\beta(c))\right|}\right)^{\frac{t}{2} n} 2\left(2^{t Z}+1\right) \\
& <C_{3}^{-t}
\end{aligned}
$$


and that for every $t$ in $\left(t_{0}, t_{*}\right)$ we have

$$
\begin{aligned}
& \sum_{k=0}^{+\infty} \exp \left(-(n+3 k) p^{+}(t)\right)\left|D f_{c}^{n+3 k}(c)\right|^{-\frac{t}{2}} \\
& =\sum_{k=0}^{+\infty} \exp \left(-(n+3 k)\left(-t \frac{\chi_{\mathrm{crit}}(c)}{2}+\delta^{+}(t)\right)\right)\left|D f_{c}^{n+3 k}(c)\right|^{-\frac{t}{2}} \\
& \leq C_{2}^{t} \exp \left(-n \delta^{+}(t)\right)\left(\frac{\exp \left(\chi_{\mathrm{crit}}(c)\right)}{\left|D f_{c}(\beta(c))\right|}\right)^{\frac{t}{2} n} \Pi\left(t \frac{\log \theta(c)}{\log 2}, \frac{3 \delta^{+}(t)}{\log 2}\right) \\
& \leq C_{2}^{t} \exp \left(-n \delta^{+}(t)\right)\left(\frac{\exp \left(\chi_{\mathrm{crit}}(c)\right)}{\left|D f_{c}(\beta(c))\right|}\right)^{\frac{t}{2} n}\left(10 \cdot 2^{t \frac{\log \theta(c)}{\log 2} \xi}+101\right) \\
& \leq C_{2}^{t} \exp \left(-n \delta^{+}(t)\right)\left(\frac{\exp \left(\chi_{\mathrm{crit}}(c)\right)}{\left|D f_{c}(\beta(c))\right|}\right)^{\frac{t}{2} n}\left(10 \cdot 2^{t Z}+101\right) \\
& <C_{3}^{-t} .
\end{aligned}
$$

Since for $t>t_{0}$ we have $p^{+}(t) \geq-\frac{t}{2} \chi_{\text {crit }}(c)$, applying part 2 of Proposition 5.6 we deduce that for $t>t_{0}$ we have $P_{c}^{\mathbb{C}}(t) \leq p^{+}(t)$ and $\mathscr{P}_{c}^{\mathbb{C}}\left(t,-t \frac{\chi_{\text {crit }}(c)}{2}\right)<0$.

To prove the assertions concerning conformal measures, recall that we have proved that for $t \geq t_{*}$ we have

$$
P_{c}^{\mathbb{R}}(t)=P_{c}^{\mathbb{C}}(t)=-t \chi_{\mathrm{crit}}(c) / 2
$$

and $\mathscr{P}_{c}^{\mathbb{C}}\left(t,-t \frac{\chi_{\text {crit }}(c)}{2}\right)<0$. This implies that for $p \geq-t \chi_{\text {crit }}(c) / 2$ we have

$$
\mathscr{P}_{c}^{\mathbb{R}}(t, p) \leq \mathscr{P}_{c}^{\mathbb{C}}(t, p) \leq \mathscr{P}_{c}^{\mathbb{C}}\left(t,-t \frac{\chi_{\text {crit }}(c)}{2}\right)<0 .
$$

So the assertions about conformal measures follow from Proposition 5.1 and Lemma 5.8.

To prove the assertions about equilibrium states, let $t \geq t_{*}$ be given and suppose by contradiction there is an equilibrium state $\rho$ of $\left.f_{c}\right|_{I_{c}}$ (resp. $f_{c}$ ) for the potential $-t \log \left|D f_{c}\right|$. Since $f_{c}$ satisfies the Collet-Eckmann condition, it follows that the Lyapunov exponent of $\rho$ is strictly positive, see [NS98, Theorem A] or [RL12, Main Theorem] for the real case and [PRLS03, Main Theorem] for the complex case. Then [Dob13, Theorem 6] in the real case and Dob12, Theorem 8] in the complex case imply that $\rho$ is absolutely continuous with respect to the $\left(t,-t \chi_{\text {crit }}(c) / 2\right)$-conformal measure for $\left.f_{c}\right|_{I_{c}}$ (resp. $f_{c}$ ) that is supported on $I_{c}$ (resp. $J_{c}$ ). This implies in particular that $\rho$ is supported on the backward orbit of $z=0$ and hence that $\rho$ charges $z=0$. This is impossible because this point is not periodic. This contradiction shows that there is no equilibrium state of $\left.f_{c}\right|_{I_{c}}$ (resp. $f_{c}$ ) for the potential $-t \log \left|D f_{c}\right|$ and completes the proof of the Main Technical Theorem.

\section{REFERENCES}

[BK98] Henk Bruin and Gerhard Keller. Equilibrium states for $S$-unimodal maps. Ergodic Theory Dynam. Systems, 18(4):765-789, 1998.

[BKNvS96] H. Bruin, G. Keller, T. Nowicki, and S. van Strien. Wild Cantor attractors exist. Ann. of Math. (2), 143(1):97-130, 1996.

[BMS03] I. Binder, N. Makarov, and S. Smirnov. Harmonic measure and polynomial Julia sets. Duke Math. J., 117(2):343-365, 2003. 
[Bow75] Rufus Bowen. Equilibrium states and the ergodic theory of Anosov diffeomorphisms. Lecture Notes in Mathematics, Vol. 470. Springer-Verlag, Berlin, 1975.

[BT06] Henk Bruin and Mike Todd. Complex maps without invariant densities. Nonlinearity, 19(12):2929-2945, 2006.

[BT09] Henk Bruin and Mike Todd. Equilibrium states for interval maps: the potential $-t \log |D f|$. Ann. Sci. Éc. Norm. Supér. (4), 42(4):559-600, 2009.

[BT12] Henk Bruin and Mike Todd. Wild attractors and thermodynamic formalism. arXiv:1202.1770 $1,2012$.

[CG93] Lennart Carleson and Theodore W. Gamelin. Complex dynamics. Universitext: Tracts in Mathematics. Springer-Verlag, New York, 1993.

[CRL10] María Isabel Cortez and Juan Rivera-Letelier. Choquet simplices as spaces of invariant probability measures on post-critical sets. Ann. Inst. H. Poincaré Anal. Non Linéaire, 27(1):95-115, 2010.

[CRL12] Daniel Coronel and Juan Rivera-Letelier. Low-temperature phase transitions in the quadratic family. 2012. arXiv:1205.1833 1.

[DGR11] Lorenzo J. Díaz, Katrin Gelfert, and Michał Rams. Rich phase transitions in step skew products. Nonlinearity, 24(12):3391-3412, 2011.

[DGR13] L.J. Díaz, K. Gelfert, and M. Rams. Abundant rich phase transitions in step skew products. arXiv:1303.0581 v1, 2013.

[DH84] A. Douady and J. H. Hubbard. Étude dynamique des polynômes complexes. Partie I, volume 84 of Publications Mathématiques d'Orsay [Mathematical Publications of Orsay]. Université de Paris-Sud, Département de Mathématiques, Orsay, 1984.

[dMvS93] Welington de Melo and Sebastian van Strien. One-dimensional dynamics, volume 25 of Ergebnisse der Mathematik und ihrer Grenzgebiete (3) [Results in Mathematics and Related Areas (3)]. Springer-Verlag, Berlin, 1993.

[Dob12] Neil Dobbs. Measures with positive Lyapunov exponent and conformal measures in rational dynamics. Trans. Amer. Math. Soc., 364(6):2803-2824, 2012.

[Dob13] Neil Dobbs. Pesin theory and equilibrium measures on the interval. arXiv:1304.3305v1, 2013.

[GPR10] Katrin Gelfert, Feliks Przytycki, and Michał Rams. On the Lyapunov spectrum for rational maps. Math. Ann., 348(4):965-1004, 2010.

[IRRL12] Irene Inoquio-Renteria and Juan Rivera-Letelier. A characterization of hyperbolic potentials of rational maps. Bull. Braz. Math. Soc. (N.S.), 43(1):99-127, 2012.

[IT11] Godofredo Iommi and Mike Todd. Dimension theory for multimodal maps. Ann. Henri Poincaré, 12(3):591-620, 2011.

[KN92] Gerhard Keller and Tomasz Nowicki. Spectral theory, zeta functions and the distribution of periodic points for Collet-Eckmann maps. Comm. Math. Phys., 149(1):31-69, 1992.

[Lop93] A. O. Lopes. The zeta function, nondifferentiability of pressure, and the critical exponent of transition. Adv. Math., 101(2):133-165, 1993.

[LOR11] R. Leplaideur, K. Oliveira, and I. Rios. Equilibrium states for partially hyperbolic horseshoes. Ergodic Theory Dynam. Systems, 31(1):179-195, 2011.

[Lyu94] Mikhail Lyubich. Combinatorics, geometry and attractors of quasi-quadratic maps. Ann. of Math. (2), 140(2):347-404, 1994.

[Mañ93] Ricardo Mañé. On a theorem of Fatou. Bol. Soc. Brasil. Mat. (N.S.), 24(1):1-11, 1993.

[Mil00] John Milnor. Periodic orbits, externals rays and the Mandelbrot set: an expository account. Astérisque, (261):xiii, 277-333, 2000. Géométrie complexe et systèmes dynamiques (Orsay, 1995).

[Mil06] John Milnor. Dynamics in one complex variable, volume 160 of Annals of Mathematics Studies. Princeton University Press, Princeton, NJ, third edition, 2006.

[Mis81] Michał Misiurewicz. Absolutely continuous measures for certain maps of an interval. Inst. Hautes Études Sci. Publ. Math., (53):17-51, 1981.

[MS00] N. Makarov and S. Smirnov. On "thermodynamics" of rational maps. I. Negative spectrum. Comm. Math. Phys., 211(3):705-743, 2000.

[MS03] N. Makarov and S. Smirnov. On thermodynamics of rational maps. II. Non-recurrent maps. J. London Math. Soc. (2), 67(2):417-432, 2003. 
[MT88] John Milnor and William Thurston. On iterated maps of the interval. In Dynamical systems (College Park, MD, 1986-87), volume 1342 of Lecture Notes in Math., pages 465-563. Springer, Berlin, 1988.

[NS98] Tomasz Nowicki and Duncan Sands. Non-uniform hyperbolicity and universal bounds for $S$-unimodal maps. Invent. Math., 132(3):633-680, 1998.

[PRL11] Feliks Przytycki and Juan Rivera-Letelier. Nice inducing schemes and the thermodynamics of rational maps. Comm. Math. Phys., 301(3):661-707, 2011.

[PRL13] Feliks Przytycki and Juan Rivera-Letelier. 2013. Manuscript in preparation.

[PRLS03] Feliks Przytycki, Juan Rivera-Letelier, and Stanislav Smirnov. Equivalence and topological invariance of conditions for non-uniform hyperbolicity in the iteration of rational maps. Invent. Math., 151(1):29-63, 2003.

[PRLS04] Feliks Przytycki, Juan Rivera-Letelier, and Stanislav Smirnov. Equality of pressures for rational functions. Ergodic Theory Dynam. Systems, 24(3):891-914, 2004.

[PS08] Yakov Pesin and Samuel Senti. Equilibrium measures for maps with inducing schemes. J. Mod. Dyn., 2(3):397-430, 2008.

[RL12] Juan Rivera-Letelier. Asymptotic expansion of smooth interval maps. 2012. arXiv:1204.3071v2.

[Roe00] Pascale Roesch. Holomorphic motions and puzzles (following M. Shishikura). In The Mandelbrot set, theme and variations, volume 274 of London Math. Soc. Lecture Note Ser., pages 117-131. Cambridge Univ. Press, Cambridge, 2000.

[Rue76] David Ruelle. A measure associated with axiom-A attractors. Amer. J. Math., 98(3):619-654, 1976.

[Sar11] Omri M. Sarig. Bernoulli equilibrium states for surface diffeomorphisms. J. Mod. Dyn., 5(3):593-608, 2011.

[Sin72] Ja. G. Sinal̆. Gibbs measures in ergodic theory. Uspehi Mat. Nauk, 27(4(166)):21-64, 1972.

[Urb03] Mariusz Urbański. Measures and dimensions in conformal dynamics. Bull. Amer. Math. Soc. (N.S.), 40(3):281-321 (electronic), 2003.

[UZ09] Mariusz Urbanski and Anna Zdunik. Ergodic theory for holomorphic endomorphisms of complex projective spaces. 2009.

[VV10] Paulo Varandas and Marcelo Viana. Existence, uniqueness and stability of equilibrium states for non-uniformly expanding maps. Ann. Inst. H. Poincaré Anal. Non Linéaire, 27(2):555-593, 2010.

Daniel Coronel, Departamento de Matemáticas, Universidad Andrés Bello, Avenida República 220, Segundo Piso, Santiago, Chile

E-mail address: alvaro.coronel@unab.cl

Juan Rivera-Letelier, Facultad de Matemáticas, Pontifica Universidad Católica de Chile, Avenida Vicuña Mackenna 4860, Santiago, Chile

E-mail address: riveraletelier@mat.puc.cl 